A N N A LES

UNIVERSITATIS MARIAE CURIE-SKŁODOWSKA

L UBLIN - P OLONIA

VOL. LXIX, NO. 1, 2015

SECTIO A

$23-57$

\author{
MARCIN BORYC and ŁUKASZ KRUK
}

\title{
A multidimensional singular stochastic control problem on a finite time horizon
}

\begin{abstract}
A singular stochastic control problem in $n$ dimensions with timedependent coefficients on a finite time horizon is considered. We show that the value function for this problem is a generalized solution of the corresponding HJB equation with locally bounded second derivatives with respect to the space variables and the first derivative with respect to time. Moreover, we prove that an optimal control exists and is unique.
\end{abstract}

Singular stochastic control is a class of problems in which one is allowed to change the drift of a Markov process (usually a diffusion) at a price proportional to the variation of the control used. Admissible controls do not have to be absolutely continuous with respect to the Lebesgue measure and they may have jumps. This setup is natural for many problems of practical interest, including portfolio selection in finance, control of queueing networks and spacecraft control, to mention just a few examples. The reader is referred to Chapter VIII of [5] for more information and basic references.

One-dimensional singular stochastic control problems are well understood by now, see, e.g., [2] and the references given there. In this case, if the running cost is convex, the optimal control makes the underlying process a reflected diffusion at the boundary of the so-called nonaction region $\mathcal{C}$. In the case of a diffusion with time-independent coefficients and discounted cost on the infinite time horizon, $\mathcal{C}$ is just an interval and the value function enjoys

2010 Mathematics Subject Classification. Primary: 93E20; Secondary: 35Q93.

Key words and phrases. Singular stochastic control, generalized derivative, HJB equation, optimal control. 
$C^{2}$-regularity (smooth fit). Both $C^{2}$-regularity of the value function and the characterization of the optimally controlled process have been extended to the case of singular control for the two-dimensional Brownian motion [14]. In $n \geq 3$ dimensions, except for "close to one-dimensional" cases of a single push direction $[15,16]$ and the radially symmetric running cost [9], only partial results are known. For example, for optimal control of the Brownian motion on the infinite time horizon, regularity of the boundary of $\mathcal{C}$ away from some "corner points" was shown in [17] and a characterization of the optimal control as a solution of the corresponding modified Skorokhod problem was given in [8].

In this paper we consider a $n$-dimensional singular stochastic control problem on a finite time horizon in which state is governed by a linear stochastic differential equation with time-dependent coefficients, the running cost is convex and controls may act in any direction. We provide estimates for the corresponding value function. These estimates imply that the value function has locally bounded generalized derivatives of the second order with respect to the space variable and of the first order with respect to the time variable. These properties are needed to consider the value function as a solution of the corresponding parabolic Hamilton-Jacobi-Bellman (HJB) equation in some generalized sense and to show existence and uniqueness of an optimal control.

Similar results have been shown in Theorem 2.1 and Theorem 3.4 from [2] in the one-dimensional case with a single push direction. The corresponding results for a multidimensional singular stochastic control problem on the infinite time horizon with time-independent drift, covariance, cost (i.e., for the elliptic case) can be found in [11]. Our article contains a generalization (or adjustment) of the approach of $[2,11]$ to an $n$-dimensional parabolic problem. It turns out that while the main ideas from those papers may be applied in our case, a mathematically rigorous analysis of our problem is somewhat delicate and needs rather careful arguments.

Our motivation for pursuing this project is the hope that the results given here will allow for a characterization of the optimal policy in the parabolic case as a solution to the corresponding Skorokhod problem for a domain with time-dependent (moving) boundary, which would be an analog of the main theorem from [8]. Indeed, the analysis of [8] used the results from [11] as the starting point, so it is plausible that their analogs will be useful in proving the corresponding result on a finite time horizon. Such a characterization would address a long-standing open problem on the structure of the optimal control in the case under consideration. We hope to address this issue in a subsequent paper.

Existence results for multidimensional singular control problems closely related to our work may be found in $[1,3,6]$. Apparently, in spite of their considerable generality, none of them contains our existence result 
as a special case. Indeed, in these papers optimal weak solutions to the corresponding SDEs are constructed, while we are concerned about finding an optimal strong solution, i.e., for the given (as opposed to some) filtration and underlying Brownian motion. Moreover, the problem considered in [1] is elliptic and the allowable control directions lie in a cone, the opening of which cannot be too wide. In $[3,6]$ the time horizon is finite, but the problem considered in [3] has the final cost instead of the running cost, while in [6] the drift of the controlled diffusion is bounded, which excludes its linear dependence on the state.

The structure of this paper is as follows. In Section 1 we pose the singular stochastic control problem, give definitions and prove lemmas needed in further considerations. In Section 2 we prove estimates for the value function. In Section 3 we consider the Bellman's dynamic programming principle (DPP) and the HJB equation related to this problem. Section 4 contains proofs of existence and uniqueness of an optimal control.

1. Notation, assumptions and lemmas. Let $\mathbb{M}^{n \times n}$ denote the set of matrices of dimension $n \times n$ with the operator norm, i.e. $\|A\|=\sup \{|A x|$ : $\left.x \in \mathbb{R}^{n},|x|=1\right\}$. Let $T>0$ be a fixed number representing our time horizon. For a function $u=u(x, t): \mathbb{R}^{n} \times[0, T] \rightarrow \mathbb{R}$ we denote the gradient and the Hessian of $u$ with respect to the space variables (i.e., $x_{i}$ ) by $D u$ and $D^{2} u$, respectively.

Let $\left(W_{t}, t \geq 0\right)$ be a standard $n$-dimensional Brownian motion defined on a complete probability space $(\Omega, \mathcal{F}, P)$. Let $\left(\mathcal{F}_{t}, t \geq 0\right)$ be the augmentation of the filtration generated by $W$ (see [7], p. 89). Denote by $\mathcal{V}$ the set of controls $v$ which are left-continuous, adapted to the filtration $\left(\mathcal{F}_{t}, t \geq 0\right)$ random processes acting from $[0, T]$ into $\mathbb{R}^{n}$, with $P$-a.s. bounded variation and s.t. $v(0)=0 P$-a.s. We note that these processes are also progressively measurable (see [7], Th. 1.1.13). As it is customary in singular stochastic control theory (see, e.g., [8]), we write $v(t)=\int_{0}^{t} \gamma(s) d \xi(s)$, where $|\gamma(t)|=1$ for every $t \in[0, T]$ and $\xi$ is nondecreasing and left-continuous. In other words, $\xi(t)$ is the total variation of $v$ on the time interval $[0, t]$ and $\gamma(t)$ is the Radon-Nikodym derivative of the vector-valued measure induced by $v$ on $[0, T]$ with respect to its total variation $\xi$.

Consider the state process described by the stochastic integral equation

$$
y_{x t}(s)=x+\int_{t}^{s}\left(a(r) y_{x t}(r)+b(r)\right) d r+\int_{t}^{s} \sigma(r) d W_{r-t}+v(s-t),
$$

$s \in[t, T]$, where $t \in[0, T]$ is an initial time, $x \in \mathbb{R}^{n}$ is an initial position, $b:[0, T] \rightarrow \mathbb{R}^{n}$ and $a, \sigma:[0, T] \rightarrow \mathbb{M}^{n \times n}$ stand for the drift and the covariance terms. Note that $\left(y_{x t}(s)\right)_{s \in[t, T]}$ is a random process adapted to $\left(\mathcal{F}_{s-t}\right)_{s \in[t, T]}$. 
To each control $v \in \mathcal{V}$, we associate a cost given by the payoff functional (2) $J_{x t}(v)=\mathbb{E}\left\{\int_{t}^{T} f\left(y_{x t}(s), s\right) e^{-\int_{t}^{s} \alpha(r) d r} d s+\int_{t}^{T} c(s) e^{-\int_{t}^{s} \alpha(r) d r} d \xi(s-t)\right\}$, where $f, \alpha$ and $c$ are respectively the running cost, the discount factor and the instantaneous cost per unit of "fuel".

Our purpose is to characterize the optimal cost, the so-called value function

$$
u(x, t)=\inf \left\{J_{x t}(v): v \in \mathcal{V}\right\} .
$$

It is often convenient to consider the following penalized problem associated with (3):

$$
u_{\epsilon}(x, t)=\inf \left\{J_{x t}(v): v \in \mathcal{V}_{\epsilon}\right\},
$$

where $\epsilon>0$ and $\mathcal{V}_{\epsilon}$ is the set of all controls $v \in \mathcal{V}$ which are Lipschitz continuous and $\left|\frac{d v}{d t}(t)\right| \leq \frac{1}{\epsilon}$ for almost every $t \in[0, T]$ almost surely.

Definition 1.1. We say that the finite time horizon stochastic control problem has the dynamic programming property in the weak sense if for every $x \in \mathbb{R}^{n}, t, t^{\prime} \in[0, T]$ s.t. $t<t^{\prime}$ and $y_{x t}^{0}(s)$ given by (1) with $v \equiv 0$ we have

$$
u(x, t) \leq \mathbb{E}\left\{\int_{t}^{t^{\prime}} f\left(y_{x t}^{0}(s), s\right) e^{-\int_{t}^{s} \alpha(r) d r} d s+u\left(y_{x t}^{0}\left(t^{\prime}\right), t^{\prime}\right) e^{-\int_{t}^{t^{\prime}} \alpha(r) d r}\right\} .
$$

Let us assume the following:

- $\alpha, c$ are Lipschitz continuous from $[0, T]$ into $[0, \infty)$ with constant $L>0$,

- $b$ is Lipschitz continuous from $[0, T]$ into $\mathbb{R}^{n}$ with the same constant $L>0$,

- $a, \sigma$ are Lipschitz continuous from $[0, T]$ into $\mathbb{M}^{n \times n}$ with the same constant $L>0$,

- there exists $c_{0}>0$ such that $c(t) \geq c_{0}$ for all $t \in[0, T]$,

- $f: \mathbb{R}^{n} \times[0, T] \rightarrow[0, \infty)$ and there exist constants $p>1, C_{0}, \tilde{C}_{0}>0$ such that for all $t, t^{\prime} \in[0, T], x, x^{\prime} \in \mathbb{R}^{n}$ and $\lambda \in(0,1)$ we have

$$
\begin{gathered}
\tilde{C}_{0}|x|^{p}-C_{0} \leq f(x, t) \leq C_{0}\left(1+|x|^{p}\right), \\
\left|f(x, t)-f\left(x+x^{\prime}, t\right)\right| \leq C_{0}\left(1+f(x, t)+f\left(x+x^{\prime}, t\right)\right)^{1-1 / p}\left|x^{\prime}\right|, \\
\left|f(x, t)-f\left(x, t^{\prime}\right)\right| \leq C_{0}\left(1+|x|^{p}\right)\left|t-t^{\prime}\right|, \\
0<f\left(x+\lambda x^{\prime}, t\right)-2 f(x, t)+f\left(x-\lambda x^{\prime}, t\right) \\
\leq C_{0} \lambda^{2}(1+f(x, t))^{q}, q=(1-2 / p)^{+} .
\end{gathered}
$$

The last assumption implies strict convexity of the function $f$ with respect to $x$. 
Let us denote by $c_{\max }$ and $\alpha_{\max }$ the maximum of the function $c, \alpha$, respectively. Moreover, by $a_{\max }, \sigma_{\max }, \beta_{\max }$ and $b_{\max }$ we denote the maximum over $t \in[0, T]$ of the norms of the matrices $a(t), \sigma(t), \beta(t)$ and the vector $b(t)$ respectively, where $\beta(t)=\sigma(t) \sigma^{T}(t)$.

Now we give lemmas needed for the proofs of the Theorems 2.1 and 2.2. The first one is well known.

Lemma 1.2. For all $x, y \geq 0$ we have

$$
\begin{gathered}
x^{p}+y^{p} \leq(x+y)^{p} \leq 2^{p-1}\left(x^{p}+y^{p}\right), \text { if } p \geq 1, \\
2^{p-1}\left(x^{p}+y^{p}\right) \leq(x+y)^{p} \leq x^{p}+y^{p}, \text { if } p \in(0,1) .
\end{gathered}
$$

Lemma 1.3 (See [10], Corollary 2.5.12). Consider an n-dimensional process described by a stochastic integral equation

$$
x(t)=x_{0}+\int_{0}^{t} g(x(s), s) d s+\int_{0}^{t} h(x(s), s) d W_{s}, \quad t \geq 0,
$$

where $x_{0} \in \mathbb{R}^{n}, g: \mathbb{R}^{n} \times[0, \infty) \rightarrow \mathbb{R}^{n}$ and $h: \mathbb{R}^{n} \times[0, \infty) \rightarrow \mathbb{M}^{n \times n}$. We assume that there exists a constant $C$ such that for all $x \in \mathbb{R}^{n}$ and $t \geq 0$

$$
|| h(x, t)||+|g(x, t)| \leq C(1+|x|) .
$$

Then for every $q>0$ there exists a constant $C_{11}>0$ depending only on $q, C$ such that for all $t \geq 0$

$$
\mathbb{E} \sup _{0 \leq s \leq t}|x(s)|^{q} \leq C_{11} e^{C_{11} t}\left(1+\left|x_{0}\right|\right)^{q} .
$$

Remark 1.4. For the process $y_{x t}$ defined by (1) with $v \equiv 0$ the assumption (10) holds. Indeed, $\sigma$ is Lipschitz continuous, independent of $x$ and defined on a finite time interval $[0, T]$, so it is bounded. We conclude the same about $a, b$, so $|g(x, t)|=|a(t) \cdot x+b(t)| \leq C(1+|x|)$, where $C=\max \{|| a(t)||,|b(t)|$ : $t \in[0, T]\}$.

Lemma 1.5. Let $x, x^{\prime} \in \mathbb{R}^{n}, t \in[0, T]$ and $g(s)=y_{x t}(s)-y_{x^{\prime} t}(s)$ for $s \in[t, T]$. Then

$$
\frac{d g}{d s}(s)=a(s) g(s), \quad|g(s)| \leq C_{12}\left|x-x^{\prime}\right|, \quad s \in[t, T],
$$

where $C_{12}=\left(1+a_{\max } T e^{a_{\max } T}\right)$.

Proof. In view of (1) we have

$$
g(s)=x-x^{\prime}+\int_{t}^{s} a(r)\left(y_{x t}(r)-y_{x^{\prime} t}(r)\right) d r=x-x^{\prime}+\int_{t}^{s} a(r) g(r) d r .
$$

Taking the derivative $d / d s$ of both sides, we get the differential equation $\frac{d g}{d s}(s)=a(s) g(s)$ with initial data $g(t)=x-x^{\prime}$. The solution of this problem satisfies

$$
|g(s)| \leq\left|x-x^{\prime}\right|+\int_{t}^{s}|a(r) g(r)| d r \leq\left|x-x^{\prime}\right|+a_{\max } \int_{t}^{s}|g(r)| d r .
$$


Using the Gronwall's inequality (see [4], p. 625), we get the second part of (12).

Lemma 1.6. Suppose that for some $x \in \mathbb{R}^{n}, t \in[0, T], v \in \mathcal{V}$ we have

$$
\mathbb{E} \int_{t}^{T} f\left(y_{x t}(s), s\right) e^{-\int_{t}^{s} \alpha(r) d r} d s \leq C\left(1+|x|^{p}\right)
$$

for a suitable constant $C>0$ independent of $x, t$. Then

$$
\mathbb{E} \int_{t}^{T} f\left(y_{x t}(s), s\right) d s \leq C_{13}\left(1+|x|^{p}\right), \text { where } C_{13}=C \cdot e^{\int_{0}^{T} \alpha(r) d r} .
$$

Proof. Indeed, multiplying both sides of our assumption by $e^{\int_{t}^{T} \alpha(r) d r}$, we get

$$
\mathbb{E} \int_{t}^{T} f\left(y_{x t}(s), s\right) e^{\int_{s}^{T} \alpha(r) d r} d s \leq C e^{\int_{t}^{T} \alpha(r) d r}\left(1+|x|^{p}\right) \leq C_{13}\left(1+|x|^{p}\right) .
$$

Of course, the left-hand side is not smaller than $\mathbb{E} \int_{t}^{T} f\left(y_{x t}(s), s\right) d s$.

Lemma 1.7 (Compare a statement in [17], p. 181). The function $J_{x t}(v)$ is convex with respect to $(x, v)$, more precisely, for all $x_{1}, x_{2} \in \mathbb{R}^{n}, t \in[0, T]$, $v_{1}, v_{2} \in \mathcal{V}$ and $\theta \in[0,1]$,

$$
J_{\theta x_{1}+(1-\theta) x_{2}, t}\left(\theta v_{1}+(1-\theta) v_{2}\right) \leq \theta J_{x_{1}, t}\left(v_{1}\right)+(1-\theta) J_{x_{2}, t}\left(v_{2}\right) .
$$

Proof. First, we note that the set $\mathcal{V}$ is obviously convex. Let $y_{x t}^{v}(s)$ be the solution of (1) corresponding to a control $v$. Denote $v_{0}=\theta v_{1}+(1-\theta) v_{2}$ and $x_{0}=\theta x_{1}+(1-\theta) x_{2}$. In view of the definition of $J_{x t}(v)$, it suffices to prove two following inequalities

$$
\begin{gathered}
f\left(y_{x_{0}, t}^{v_{0}}(s), s\right) \leq \theta f\left(y_{x_{1}, t}^{v_{1}}(s), s\right)+(1-\theta) f\left(y_{x_{2}, t}^{v_{2}}(s), s\right), s \in[t, T], \\
\int_{t}^{T} d \xi_{0}(s-t) \leq \theta \int_{t}^{T} d \xi_{1}(s-t)+(1-\theta) \int_{t}^{T} d \xi_{2}(s-t),
\end{gathered}
$$

where $\xi_{0}, \xi_{1}, \xi_{2}$ are the total variations of $v_{0}, v_{1}, v_{2}$ respectively.

The latter inequality is a consequence of the fact that the variation of the sum of functions is not greater than the sum of their variations. So $\xi_{0} \leq$ $\theta \xi_{1}+(1-\theta) \xi_{2}$. Because $\xi_{0}, \xi_{1}, \xi_{2}$ are nondecreasing and $\xi_{0}(0)=\xi_{1}(0)=$ $\xi_{2}(0)=0 P$-a.s., we conclude that $(15)$ is true.

To prove (14) we show first that

$$
y_{x_{0}, t}^{v_{0}}(s)=\theta y_{x_{1}, t}^{v_{1}}(s)+(1-\theta) y_{x_{2}, t}^{v_{2}}(s) .
$$

Indeed, using (1) we get

$$
y_{x_{i}, t}^{v_{i}}(s)=x_{i}+\int_{t}^{s}\left(a(r) y_{x_{i}, t}^{v_{i}}(r)+b(r)\right) d r+\int_{t}^{s} \sigma(r) d W_{r-t}+v_{i}(s-t),
$$


$i=0,1,2$. Let $g(s)=y_{x_{0}, t}^{v_{0}}(s)-\theta y_{x_{1}, t}^{v_{1}}(s)-(1-\theta) y_{x_{2}, t}^{v_{2}}(s)$. Then

$$
g(s)=\int_{t}^{s} a(r)\left(y_{x_{0}, t}^{v_{0}}(r)-\theta y_{x_{1}, t}^{v_{1}}(r)-(1-\theta) y_{x_{2}, t}^{v_{2}}(r)\right) d r=\int_{t}^{s} a(r) g(r) d r .
$$

Taking the derivative $d / d s$ of both sides, we get the differential equation $\frac{d g}{d s}(s)=a(s) g(s)$ with initial data $g(t)=x_{0}-\theta x_{1}-(1-\theta) x_{2}=0$. The solution of this problem is $g(s) \equiv 0$, so (16) holds. Using (16) and convexity of $f$ we have (14).

Lemma 1.8. Suppose that for some $t^{\prime} \in[0, T], x \in \mathbb{R}^{n}, v \in \mathcal{V}$ we have

$$
\mathbb{E} \int_{0}^{T-t^{\prime}} c\left(t^{\prime}+s\right) e^{-\int_{0}^{s} \alpha\left(t^{\prime}+r\right) d r} d \xi(s) \leq C\left(1+|x|^{p}\right)
$$

for a suitable constant $C>0$ independent of $x, t^{\prime}$. Then there exists a constant $C_{17}>0$ independent of $x, t^{\prime}$ such that

$$
\mathbb{E} \xi\left(T-t^{\prime}\right) \leq C_{17}\left(1+|x|^{p}\right)
$$

Proof. Indeed, multiplying both sides of our assumption by $e^{\int_{0}^{T-t^{\prime}} \alpha\left(t^{\prime}+r\right) d r}$ and using the lower bound of $c$, we get

$$
\begin{aligned}
c_{0} \mathbb{E} \xi\left(T-t^{\prime}\right) & =c_{0} \mathbb{E} \int_{0}^{T-t^{\prime}} d \xi(s) \leq \mathbb{E} \int_{0}^{T-t^{\prime}} c\left(t^{\prime}+s\right) e^{\int_{s}^{T-t^{\prime}} \alpha\left(t^{\prime}+r\right) d r} d \xi(s) \\
& \leq C e^{\int_{0}^{T} \alpha(r) d r}\left(1+|x|^{p}\right)
\end{aligned}
$$

Lemma 1.9. Suppose that for some $x \in \mathbb{R}^{n}, t \in[0, T], v \in \mathcal{V}$ we have

$$
\mathbb{E} \int_{0}^{T-t} f\left(y_{x t}(t+s), t+s\right) e^{-\int_{0}^{s} \alpha(t+r) d r} d s \leq C\left(1+|x|^{p}\right)
$$

for a suitable constant $C>0$ independent of $x, t$. Then there exists a constant $C_{18}>0$ independent of $x, t$ such that

$$
\mathbb{E} \int_{0}^{T-t}\left(1+\left|y_{x t}(t+s)\right|^{p}\right) d s \leq C_{18}\left(1+|x|^{p}\right)
$$

Proof. From Lemma 1.6 we know that

$$
\mathbb{E} \int_{0}^{T-t} f\left(y_{x t}(t+s), t+s\right) d s \leq C_{13}\left(1+|x|^{p}\right) .
$$

Using (6), we get

$$
\mathbb{E} \int_{0}^{T-t}\left(\tilde{C}_{0}\left|y_{x t}(t+s)\right|^{p}-C_{0}\right) d s \leq C_{13}\left(1+|x|^{p}\right) .
$$

Hence

$$
\tilde{C}_{0} \mathbb{E} \int_{0}^{T-t}\left|y_{x t}(t+s)\right|^{p} d s \leq\left(C_{13}+C_{0} T\right)\left(1+|x|^{p}\right)
$$


and finally

$$
\tilde{C}_{0} \mathbb{E} \int_{0}^{T-t}\left(1+\left|y_{x t}(t+s)\right|^{p}\right) d s \leq\left(C_{13}+C_{0} T+\tilde{C}_{0} T\right)\left(1+|x|^{p}\right) .
$$

Lemma 1.10. Let $0 \leq t^{\prime} \leq t \leq T$ and suppose that for some $x \in \mathbb{R}^{n}, v \in \mathcal{V}$ we have

$$
\mathbb{E} \int_{0}^{T-t^{\prime}} f\left(y_{x t^{\prime}}\left(t^{\prime}+s\right), t^{\prime}+s\right) e^{-\int_{0}^{s} \alpha\left(t^{\prime}+r\right) d r} d s \leq C\left(1+|x|^{p}\right)
$$

for a suitable constant $C>0$ independent of $x, t, t^{\prime}$. Then there exists a constant $C_{19}>0$ independent of $x, t, t^{\prime}$ such that

$$
\mathbb{E} \int_{0}^{T-t} f\left(y_{x t^{\prime}}\left(t^{\prime}+s\right), t+s\right) d s \leq C_{19}\left(1+|x|^{p}\right) .
$$

Proof. We observe that using (8) we have

$$
\begin{aligned}
f\left(y_{x t^{\prime}}\left(t^{\prime}+s\right), t+s\right) \leq & \left|f\left(y_{x t^{\prime}}\left(t^{\prime}+s\right), t+s\right)-f\left(y_{x t^{\prime}}\left(t^{\prime}+s\right), t^{\prime}+s\right)\right| \\
& +f\left(y_{x t^{\prime}}\left(t^{\prime}+s\right), t^{\prime}+s\right) \\
\leq & C_{0}\left|t-t^{\prime}\right|\left(1+\left|y_{x t^{\prime}}\left(t^{\prime}+s\right)\right|^{p}\right)+f\left(y_{x t^{\prime}}\left(t^{\prime}+s\right), t^{\prime}+s\right) .
\end{aligned}
$$

Hence, in view of Lemma 1.6 and Lemma 1.9, we get

$$
\begin{aligned}
\mathbb{E} \int_{0}^{T-t} f\left(y_{x t^{\prime}}\left(t^{\prime}+s\right), t+s\right) d s & \leq C_{0}\left|t-t^{\prime}\right| C_{18}\left(1+|x|^{p}\right)+C_{13}\left(1+|x|^{p}\right) \\
& \leq C_{19}\left(1+|x|^{p}\right)
\end{aligned}
$$

where $C_{19}=C_{0} T C_{18}+C_{13}$.

Lemma 1.11. Let $0 \leq t^{\prime} \leq t \leq T, x \in \mathbb{R}^{n}, v \in \mathcal{V}$. Assume that

$$
\mathbb{E} \int_{0}^{T-t^{\prime}} f\left(y_{x t^{\prime}}\left(t^{\prime}+s\right), t^{\prime}+s\right) e^{-\int_{0}^{s} \alpha\left(t^{\prime}+r\right) d r} d s \leq C\left(1+|x|^{p}\right)
$$

for a suitable constant $C>0$ independent of $x, t^{\prime}, t$. Then there exists a constant $C_{20}>0$ independent of $x, t^{\prime}, t$ such that for all $s \in[0, T-t]$ we have

$$
\mathbb{E}\left|y_{x t^{\prime}}\left(t^{\prime}+s\right)-y_{x t}(t+s)\right|^{p} \leq C_{20}\left|t-t^{\prime}\right|^{p}\left(1+|x|^{p}\right) .
$$

Proof. For $s \in[0, T-t]$, we have

$$
\begin{aligned}
& y_{x t}(t+s)=x+\int_{0}^{s}\left(a(t+r) y_{x t}(t+r)+b(t+r)\right) d r+\int_{0}^{s} \sigma(t+r) d W_{r}+v(s), \\
& y_{x t^{\prime}}\left(t^{\prime}+s\right)=x+\int_{0}^{s}\left(a\left(t^{\prime}+r\right) y_{x t^{\prime}}\left(t^{\prime}+r\right)+b\left(t^{\prime}+r\right)\right) d r+\int_{0}^{s} \sigma\left(t^{\prime}+r\right) d W_{r}+v(s),
\end{aligned}
$$

$$
y_{x t^{\prime}}\left(t^{\prime}+s\right)-y_{x t}(t+s)=A_{s}+B_{s}+M_{s}
$$


where

$$
\begin{aligned}
& A_{s}=\int_{0}^{s}\left(a\left(t^{\prime}+r\right) y_{x t^{\prime}}\left(t^{\prime}+r\right)-a(t+r) y_{x t}(t+r)\right) d r=A_{s}^{1}+A_{s}^{2} \\
& A_{s}^{1}=\int_{0}^{s} a(t+r)\left(y_{x t^{\prime}}\left(t^{\prime}+r\right)-y_{x t}(t+r)\right) d r \\
& A_{s}^{2}=\int_{0}^{s}\left(a\left(t^{\prime}+r\right)-a(t+r)\right) y_{x t^{\prime}}\left(t^{\prime}+r\right) d r \\
& B_{s}=\int_{0}^{s}\left(b\left(t^{\prime}+r\right)-b(t+r)\right) d r \\
& M_{s}=\int_{0}^{s}\left(\sigma\left(t^{\prime}+r\right)-\sigma(t+r)\right) d W_{r} .
\end{aligned}
$$

Recall that $a, b, \sigma$ are Lipschitz continuous with the constant $L$. The process $M_{s}$ is a martingale with quadratic variation

$$
[M]_{s}=\int_{0}^{s}\left(\sigma\left(t^{\prime}+r\right)-\sigma(t+r)\right)^{2} d r \leq L^{2}\left|t-t^{\prime}\right|^{2} s .
$$

This, together with the Burkholder-Davis-Gundy inequalities (see [7], Theorem 3.3.28), implies the existence of a constant $C_{p}$, depending only on $p$, such that

$$
\mathbb{E} \sup _{0 \leq s \leq T-t}\left|M_{s}\right|^{p} \leq C_{p} L^{p} T^{\frac{p}{2}}\left|t-t^{\prime}\right|^{p} .
$$

Clearly,

$$
\sup _{0 \leq s \leq T-t}\left|B_{s}\right| \leq L T\left|t-t^{\prime}\right|
$$

By the Hölder's inequality, for $q=p /(p-1)$ we have

$$
\begin{aligned}
& \left|A_{s}^{1}\right|^{p} \leq\left(a_{\max }^{q} s\right)^{\frac{p}{q}} \int_{0}^{s}\left|y_{x t^{\prime}}\left(t^{\prime}+r\right)-y_{x t}(t+r)\right|^{p} d r \\
& \left|A_{s}^{2}\right|^{p} \leq\left(\left(L\left|t-t^{\prime}\right|\right)^{q} s\right)^{\frac{p}{q}} \int_{0}^{s}\left|y_{x t^{\prime}}\left(t^{\prime}+r\right)\right|^{p} d r .
\end{aligned}
$$

By Lemma 1.9, the inequality (18) holds for every $t \in[0, T]$. Lemma 1.2 and the relations (18), (21)-(25) imply that the random variable

$$
\sup _{0 \leq s \leq T-t}\left|y_{x t^{\prime}}\left(t^{\prime}+s\right)-y_{x t}(t+s)\right|^{p}
$$

is integrable and hence, by the Lebesgue dominated convergence theorem, the function $F(s)=\mathbb{E}\left|y_{x t^{\prime}}\left(t^{\prime}+s\right)-y_{x t}(t+s)\right|^{p}$ is continuous on $[0, T-t]$. From Lemma 1.2 and (18), (21)-(25) we also have, for each $s \in[0, T-t]$,

$$
F(s) \leq c_{1}\left|t-t^{\prime}\right|^{p}\left(1+|x|^{p}\right)+c_{2} \int_{0}^{s} F(r) d r
$$


where $c_{1}=2^{2 p-2}\left(L^{p} T^{p}+C_{p} L^{p} T^{\frac{p}{2}}+(L T)^{\frac{p}{q}} C_{18}\right), c_{2}=2^{2 p-2} a_{\max }^{p} T^{\frac{p}{q}}$. This, together with the Gronwall's inequality (see, e.g., [7], Problem 5.2.7), implies that for all $s \in[0, T-t]$,

$$
\left.F(s) \leq c_{1}\left|t-t^{\prime}\right|^{p}\left(1+|x|^{p}\right)\left(1+c_{2} \int_{0}^{s} e^{c_{2}(s-r)} d r\right)\right) .
$$

We have obtained (20) with $C_{20}=c_{1}\left(1+c_{2} \int_{0}^{T} e^{c_{2}(T-r)} d r\right)$.

Lemma 1.12. Suppose that for some $x \in \mathbb{R}^{n}, t \in[0, T], v \in \mathcal{V}$ we have

$$
\mathbb{E} \int_{t}^{T} f\left(y_{x t}(s), s\right) e^{-\int_{t}^{s} \alpha(r) d r} d s \leq C\left(1+|x|^{p}\right)
$$

for a suitable constant $C>0$ independent of $x, t$. Then there exists a constant $C_{26}>0$ independent of $x, x^{\prime}, t$ such that for every $x^{\prime} \in \mathbb{R}^{n}$,

$$
\mathbb{E} \int_{t}^{T} f\left(y_{x+x^{\prime}, t}(s), s\right) d s \leq C_{26}\left(1+|x|^{p}+\left|x+x^{\prime}\right|^{p}\right) .
$$

Proof. From (6) and Lemma 1.2 we have

$$
\begin{aligned}
& \mathbb{E} \int_{t}^{T} f\left(y_{x+x^{\prime}, t}(s), s\right) d s \leq \mathbb{E} \int_{t}^{T} C_{0}\left(1+\left|y_{x+x^{\prime}, t}(s)\right|^{p}\right) d s \\
& \leq T C_{0}+C_{0} 2^{p-1} \mathbb{E} \int_{t}^{T}\left|y_{x+x^{\prime}, t}(s)-y_{x, t}(s)\right|^{p} d s+C_{0} 2^{p-1} \mathbb{E} \int_{t}^{T}\left|y_{x, t}(s)\right|^{p} d s .
\end{aligned}
$$

Now using Lemma 1.5, Lemma 1.9 and Lemma 1.2 again, we get

$$
\begin{aligned}
& \mathbb{E} \int_{t}^{T} f\left(y_{x+x^{\prime}, t}(s), s\right) d s \leq T C_{0}+C_{0} 2^{p-1} T \cdot C_{12}^{p}\left|x^{\prime}\right|^{p}+C_{0} 2^{p-1} C_{18}\left(1+|x|^{p}\right) \\
& \quad \leq T C_{0}+C_{0} 2^{2 p-2} T \cdot C_{12}^{p}\left(\left|x^{\prime}+x\right|^{p}+|x|^{p}\right)+C_{0} 2^{p-1} C_{18}\left(1+|x|^{p}\right) \\
& \quad \leq C_{26}\left(1+|x|^{p}+\left|x+x^{\prime}\right|^{p}\right),
\end{aligned}
$$

where $C_{26}=C_{0}\left(T+2^{2 p-2} T \cdot C_{12}^{p}+2^{p-1} C_{18}\right)$.

Lemma 1.13. Suppose that for some $x \in \mathbb{R}^{n}, t^{\prime} \in[0, T], v \in \mathcal{V}$ we have

$$
\mathbb{E} \int_{0}^{T-t^{\prime}} f\left(y_{x t^{\prime}}\left(t^{\prime}+s\right), t^{\prime}+s\right) e^{-\int_{0}^{s} \alpha\left(t^{\prime}+r\right) d r} d s \leq C\left(1+|x|^{p}\right)
$$

for a suitable constant $C>0$ independent of $x, t^{\prime}$. Then there exists a constant $C_{27}>0$ independent of $x, t^{\prime}, t$ such that for every $t \in\left[t^{\prime}, T\right]$,

$$
\mathbb{E} \int_{0}^{T-t} f\left(y_{x t}(t+s), t+s\right) d s \leq C_{27}\left(1+|x|^{p}\right) .
$$


Proof. Using (6) and Lemma 1.2, we have

$$
\begin{aligned}
& \mathbb{E} \int_{0}^{T-t} f\left(y_{x t}(t+s), t+s\right) d s \leq \mathbb{E} \int_{0}^{T-t} C_{0}\left(1+\left|y_{x t}(t+s)\right|^{p}\right) d s \\
& \leq C_{0} T+2^{p-1} C_{0} \mathbb{E} \int_{0}^{T-t}\left|y_{x t^{\prime}}\left(t^{\prime}+s\right)\right|^{p} d s \\
& \quad+2^{p-1} C_{0} \mathbb{E} \int_{0}^{T-t}\left|y_{x t}(t+s)-y_{x t^{\prime}}\left(t^{\prime}+s\right)\right|^{p} d s .
\end{aligned}
$$

In view of Lemma 1.9, the Fubini's theorem and Lemma 1.11, we get

$$
\begin{aligned}
& \mathbb{E} \int_{0}^{T-t} f\left(y_{x t}(t+s), t+s\right) d s \\
& \quad \leq C_{0} T+2^{p-1} C_{0} C_{18}\left(1+|x|^{p}\right)+2^{p-1} C_{0} T C_{20}\left|t-t^{\prime}\right|^{p}\left(1+|x|^{p}\right) \\
& \quad \leq C_{27}\left(1+|x|^{p}\right),
\end{aligned}
$$

where $C_{27}=C_{0}\left(T+2^{p-1} C_{18}+2^{p-1} T^{p+1} C_{20}\right)$.

The next two definitions and lemma refer to mollification of a given function (see [4], p. 629-630).

Definition 1.14. Define $\eta \in C^{\infty}\left(\mathbb{R}^{n}\right)$ by

$$
\eta(x)= \begin{cases}C_{28} \exp \left(\frac{1}{|x|^{2}-1}\right) & \text { if }|x|<1 \\ 0 & \text { if }|x| \geq 1,\end{cases}
$$

where the constant $C_{28}$ is selected so that $\int_{\mathbb{R}^{n}} \eta(x) d x=1$. For each $m \in \mathbb{N}$ set $\eta_{m}(x)=m^{n} \cdot \eta(m x)$. We call $\eta$ the standard mollifier. The functions $\eta_{m}$ belong to the class $C^{\infty}\left(\mathbb{R}^{n}\right)$ and satisfy $\int_{\mathbb{R}^{n}} \eta_{m}(x) d x=1$.

Definition 1.15. Fix $t^{\prime} \in[0, T]$. For each $m \in \mathbb{N}$ we define mollification of the function $u\left(\cdot, t^{\prime}\right)$ by

$$
u_{m}(x)=\int_{B\left(0, \frac{1}{m}\right)} \eta_{m}(y) u\left(x-y, t^{\prime}\right) d y, \quad x \in \mathbb{R}^{n},
$$

where $B(0, r)=\left\{x \in \mathbb{R}^{n}:|x|<r\right\}$.

Lemma 1.16. For each $m \in \mathbb{N}$ we have $u_{m} \in C^{\infty}\left(\mathbb{R}^{n}\right)$. Moreover, if $u\left(\cdot, t^{\prime}\right)$ is continuous, then $u_{m}(x) \rightarrow u\left(x, t^{\prime}\right)$ uniformly on compact subsets of $\mathbb{R}^{n}$ as $m \rightarrow \infty$.

2. Estimates for the value function. Let the assumptions from Section 1 appearing immediately after Definition 1.1 hold.

Theorem 2.1. Let $u$ be the value function defined by (3). Then for some positive constants $C_{29}, C_{30}, C_{31}$, the same $p>1$ as in the assumptions (6)(9) and every $t \in[0, T], x, x^{\prime} \in \mathbb{R}^{n}$ and $\lambda \in(0,1)$, the following estimates 
hold:

$$
\begin{gathered}
0 \leq u(x, t) \leq C_{29}\left(1+|x|^{p}\right) \\
\left|u(x, t)-u\left(x+x^{\prime}, t\right)\right| \leq C_{30}\left(1+|x|^{p-1}+\left|x+x^{\prime}\right|^{p-1}\right)\left|x^{\prime}\right| \\
0 \leq u\left(x+\lambda x^{\prime}, t\right)-2 u(x, t)+u\left(x-\lambda x^{\prime}, t\right) \leq C_{31} \lambda^{2}(1+|x|)^{(p-2)^{+}} .
\end{gathered}
$$

Proof: Proof of (29). Nonnegativity of $u$ is the consequence of nonnegativity of $f$ and $c$. Next, taking the control $v \equiv 0$ and using (6), the Fubini's theorem, Lemma 1.3 and Lemma 1.2, we get

$$
\begin{aligned}
u(x, t) & \leq J_{x t}(0)=\mathbb{E} \int_{t}^{T} f\left(y_{x t}^{0}(s), s\right) e^{-\int_{t}^{s} \alpha(r) d r} d s \\
& \leq \mathbb{E} \int_{t}^{T} C_{0}\left(1+\left|y_{x t}^{0}(s)\right|^{p}\right) d s=C_{0} \int_{t}^{T} \mathbb{E}\left(1+\left|y_{x t}^{0}(s)\right|^{p}\right) d s \\
& \leq C_{0} \int_{t}^{T} \mathbb{E}\left(1+C_{11} e^{C_{11}(s-t)}(1+|x|)^{p}\right) d s \\
& \leq C_{0} \int_{0}^{T}\left(1+C_{11} e^{C_{11} T} 2^{p-1}\right)\left(1+|x|^{p}\right) d s \\
& =C_{0} T\left(1+C_{11} e^{C_{11} T} 2^{p-1}\right)\left(1+|x|^{p}\right) \\
& =C_{29}\left(1+|x|^{p}\right),
\end{aligned}
$$

where $C_{29}$ depends only on $C_{0}, T, C_{11}, p$, so (29) is proved.

Proof of (30). Now we note that

$$
\begin{aligned}
u\left(x+x^{\prime}, t\right)-u(x, t) & =\inf _{v^{\prime} \in \mathcal{V}} \sup _{v \in \mathcal{V}}\left(J_{x+x^{\prime}, t}\left(v^{\prime}\right)-J_{x, t}(v)\right) \\
& \leq \sup _{v \in \mathcal{V}}\left(J_{x+x^{\prime}, t}(v)-J_{x, t}(v)\right) .
\end{aligned}
$$

Hence

$$
\begin{aligned}
u\left(x+x^{\prime}, t\right)-u(x, t) & \leq \sup _{v \in \mathcal{V}}\left|J_{x t}(v)-J_{x+x^{\prime}, t}(v)\right| \\
& \leq \sup _{v \in \mathcal{V}} \mathbb{E} \int_{t}^{T}\left|f\left(y_{x t}(s), s\right)-f\left(y_{x+x^{\prime}, t}(s), s\right)\right| e^{-\int_{t}^{s} \alpha(r) d r} d s .
\end{aligned}
$$

Applying (7), we can estimate the last expression from above by $\sup _{v \in \mathcal{V}} \mathbb{E} \int_{t}^{T} C_{0}\left(1+f\left(y_{x t}(s), s\right)+f\left(y_{x+x^{\prime}, t}(s), s\right)\right)^{1-1 / p} \cdot\left|y_{x t}(s)-y_{x+x^{\prime}, t}(s)\right| d s$.

Using Lemma 1.5, we have

$$
\begin{aligned}
& u\left(x+x^{\prime}, t\right)-u(x, t) \\
& \quad \leq \sup _{v \in \mathcal{V}} C_{0} C_{12}\left|x^{\prime}\right| \cdot \mathbb{E} \int_{t}^{T}\left(1+f\left(y_{x t}(s), s\right)+f\left(y_{x+x^{\prime}, t}(s), s\right)\right)^{\frac{p-1}{p}} d s .
\end{aligned}
$$


We use the Hölder's inequality with exponent $\frac{p}{p-1}$ to estimate the last expression above by

(32) $\sup _{v \in \mathcal{V}} C_{0} C_{12}\left|x^{\prime}\right| \cdot\left(\mathbb{E} \int_{t}^{T}\left(1+f\left(y_{x t}(s), s\right)+f\left(y_{x+x^{\prime}, t}(s), s\right)\right) d s\right)^{\frac{p-1}{p}} T^{\frac{1}{p}}$.

By virtue of (29) we can consider only those controls $v$ for which

$$
\mathbb{E} \int_{t}^{T} f\left(y_{x t}(s), s\right) e^{-\int_{t}^{s} \alpha(r) d r} d s \leq\left(C_{29}+\epsilon\right)\left(1+|x|^{p}\right)
$$

for some arbitrary $\epsilon>0$. From (32), Lemma 1.6 and Lemma 1.12 we see that

$$
\begin{aligned}
u\left(x+x^{\prime}, t\right)-u(x, t) \leq & C_{0} C_{12}\left|x^{\prime}\right|\left(T+C_{13}\left(1+|x|^{p}\right)\right. \\
& \left.+C_{26}\left(1+|x|^{p}+\left|x+x^{\prime}\right|^{p}\right)\right)^{\frac{p-1}{p}} T^{\frac{1}{p}} \\
\leq & C_{30}\left|x^{\prime}\right|\left(1+|x|^{p}+\left|x+x^{\prime}\right|^{p}\right)^{\frac{p-1}{p}},
\end{aligned}
$$

where $C_{30}=T^{1 / p} \cdot C_{0} C_{12}\left(T+C_{13}+C_{26}\right)^{1-1 / p}$. Finally using Lemma 1.2, we get

$$
u\left(x+x^{\prime}, t\right)-u(x, t) \leq C_{30}\left(1+|x|^{p-1}+\left|x+x^{\prime}\right|^{p-1}\right)\left|x^{\prime}\right| .
$$

In an analogous manner we get the same estimate for $u(x, t)-u\left(x+x^{\prime}, t\right)$.

Proof of (31). We observe that

$$
\begin{aligned}
& u\left(x+\lambda x^{\prime}, t\right)+u\left(x-\lambda x^{\prime}, t\right)-2 u(x, t) \\
& \leq \sup _{v \in \mathcal{V}}\left(J_{x+\lambda x^{\prime}, t}(v)+J_{x-\lambda x^{\prime}, t}(v)-2 J_{x t}(v)\right) \\
& =\sup _{v \in \mathcal{V}} \mathbb{E} \int_{t}^{T}\left(f\left(y_{x+\lambda x^{\prime}, t}(s), s\right)+f\left(y_{x-\lambda x^{\prime}, t}(s), s\right)-2 f\left(y_{x t}(s), s\right)\right) e^{-\int_{t}^{s} \alpha(r) d r} d s .
\end{aligned}
$$

In view of (12) we can apply (9) to get

$$
\begin{aligned}
u\left(x+\lambda x^{\prime}, t\right) & +u\left(x-\lambda x^{\prime}, t\right)-2 u(x, t) \\
& \leq \sup _{v \in \mathcal{V}} \mathbb{E} \int_{t}^{T} C_{0} \lambda^{2}\left(1+f\left(y_{x t}(s), s\right)\right)^{(1-2 / p)^{+}} d s .
\end{aligned}
$$

If $p \leq 2$ we have $u\left(x+\lambda x^{\prime}, t\right)+u\left(x-\lambda x^{\prime}, t\right)-2 u(x, t) \leq C_{0} T \lambda^{2}$. If $p>2$ we use the Hölder inequality with exponent $\frac{p}{p-2}$ to get

$$
\begin{aligned}
u\left(x+\lambda x^{\prime}, t\right) & +u\left(x-\lambda x^{\prime}, t\right)-2 u(x, t) \\
& \leq \sup _{v \in \mathcal{V}} C_{0} \lambda^{2}\left(\mathbb{E} \int_{t}^{T}\left(1+f\left(y_{x t}(s), s\right) d s\right)^{1-2 / p} T^{2 / p} .\right.
\end{aligned}
$$


By virtue of (29) we can consider only those controls $v$ for which

$$
\mathbb{E} \int_{t}^{T} f\left(y_{x t}(s), s\right) e^{-\int_{t}^{s} \alpha(r) d r} d s \leq\left(C_{29}+\epsilon\right)\left(1+|x|^{p}\right)
$$

for some arbitrary $\epsilon>0$. From Lemma 1.6 and Lemma 1.2 we see that

$$
\begin{aligned}
u\left(x+\lambda x^{\prime}, t\right)+u(x & \left.-\lambda x^{\prime}, t\right)-2 u(x, t) \\
& \leq C_{0} \lambda^{2}\left(T+C_{13}\left(1+|x|^{p}\right)\right)^{1-2 / p} T^{2 / p} \\
& \leq C_{31} \lambda^{2}\left(1+|x|^{p}\right)^{1-2 / p} \\
& \leq C_{31} \lambda^{2}(1+|x|)^{p-2},
\end{aligned}
$$

where $C_{31}=T^{2 / p} C_{0}\left(T+C_{13}\right)^{1-2 / p}$. We have proved the upper bound of (31).

To prove the lower bound of (31), it clearly suffices to prove convexity of $u(x, t)$ with respect to the first variable. In view of the definition of $u$ we know that for every $\epsilon>0, x_{1}, x_{2} \in \mathbb{R}^{n}, t \in[0, T], \theta \in[0,1]$ there exist $v_{1}, v_{2} \in \mathcal{V}$ such that $J_{x_{i}, t}\left(v_{i}\right) \leq u\left(x_{i}, t\right)+\epsilon, i=1,2$.

Using Lemma 1.7, we get

$$
\begin{aligned}
u\left(\theta x_{1}+(1-\theta) x_{2}, t\right) & \leq J_{\theta x_{1}+(1-\theta) x_{2}, t}\left(\theta v_{1}+(1-\theta) v_{2}\right) \\
& \leq \theta J_{x_{1}, t}\left(v_{1}\right)+(1-\theta) J_{x_{2}, t}\left(v_{2}\right) \\
& \leq \theta u\left(x_{1}, t\right)+(1-\theta) u\left(x_{2}, t\right)+\epsilon
\end{aligned}
$$

Because $\epsilon>0$ is arbitrary, we get convexity of $u(x, t)$ with respect to the first variable.

Theorem 2.2. Let the assumptions of Theorem 2.1 be satisfied. Assume that the dynamic programming property in the weak sense holds (Definition 1.1). Then for some constant $C_{33}>0$ and every $t, t^{\prime} \in[0, T], x \in \mathbb{R}^{n}$, we have

$$
\left|u(x, t)-u\left(x, t^{\prime}\right)\right| \leq C_{33}\left(1+|x|^{p}\right)\left|t-t^{\prime}\right| .
$$

Proof. We note that

$$
\begin{aligned}
u(x, t)-u\left(x, t^{\prime}\right) & =\inf _{v \in \mathcal{V}} \sup _{v^{\prime} \in \mathcal{V}}\left(J_{x t}(v)-J_{x t^{\prime}}\left(v^{\prime}\right)\right) \\
& \leq \sup _{v^{\prime} \in \mathcal{V}}\left(J_{x t}\left(v^{\prime}\right)-J_{x t^{\prime}}\left(v^{\prime}\right)\right) .
\end{aligned}
$$


For $t^{\prime} \leq t$ the difference $J_{x t}(v)-J_{x t^{\prime}}(v)$ is equal to

$$
\begin{aligned}
& \mathbb{E}\left\{\int _ { 0 } ^ { T - t } \left(f\left(y_{x t}(t+s), t+s\right) e^{-\int_{0}^{s} \alpha(t+r) d r}\right.\right. \\
& \left.\quad-f\left(y_{x t^{\prime}}\left(t^{\prime}+s\right), t^{\prime}+s\right) e^{-\int_{0}^{s} \alpha\left(t^{\prime}+r\right) d r}\right) d s \\
& \quad+\int_{0}^{T-t}\left(c(t+s) e^{-\int_{0}^{s} \alpha(t+r) d r}-c\left(t^{\prime}+s\right) e^{-\int_{0}^{s} \alpha\left(t^{\prime}+r\right) d r}\right) d \xi(s) \\
& \quad-\int_{T-t}^{T-t^{\prime}} f\left(y_{x t^{\prime}}\left(t^{\prime}+s\right), t^{\prime}+s\right) e^{-\int_{0}^{s} \alpha\left(t^{\prime}+r\right) d r} d s \\
& \left.-\int_{T-t}^{T-t^{\prime}} c\left(t^{\prime}+s\right) e^{-\int_{0}^{s} \alpha\left(t^{\prime}+r\right) d r} d \xi(s)\right\} .
\end{aligned}
$$

Let us denote the expectations of the first two integrals in the last expression by $A$ and $B$, respectively. Because the last two integrals are nonnegative we get

$$
J_{x t}(v)-J_{x t^{\prime}}(v) \leq A+B .
$$

We can estimate $B$ as follows:

$$
B \leq \mathbb{E} \int_{0}^{T-t}\left|c(t+s) e^{-\int_{0}^{s} \alpha(t+r) d r}-c\left(t^{\prime}+s\right) e^{-\int_{0}^{s} \alpha\left(t^{\prime}+r\right) d r}\right| d \xi(s) .
$$

Adding and subtracting $c(t+s) e^{-\int_{0}^{s} \alpha\left(t^{\prime}+r\right) d r}$ under the absolute value sign and using the triangle inequality and positivity of $\alpha$, we get

$B \leq \mathbb{E} \int_{0}^{T-t}\left(c_{\max }\left|e^{-\int_{0}^{s} \alpha(t+r) d r}-e^{-\int_{0}^{s} \alpha\left(t^{\prime}+r\right) d r}\right|+\left|c(t+s)-c\left(t^{\prime}+s\right)\right|\right) d \xi(s)$.

Because $\left|e^{x}-e^{y}\right| \leq|x-y|$ for $x, y \leq 0$ and $c, \alpha$ are Lipschitz continuous, we have

$$
\begin{aligned}
B & \leq \mathbb{E} \int_{0}^{T-t}\left(c_{\max } \int_{0}^{s}\left|\alpha(t+r)-\alpha\left(t^{\prime}+r\right)\right| d r+\left|c(t+s)-c\left(t^{\prime}+s\right)\right|\right) d \xi(s) \\
& \leq\left(c_{\max } T+1\right) L\left|t-t^{\prime}\right| \mathbb{E} \int_{0}^{T-t} d \xi(s)=\left(c_{\max } T+1\right) L\left|t-t^{\prime}\right| \mathbb{E} \xi(T-t) .
\end{aligned}
$$

By virtue of (29) we can consider only those controls $v$ for which

$$
\mathbb{E} \int_{0}^{T-t^{\prime}} c\left(t^{\prime}+s\right) e^{-\int_{0}^{s} \alpha\left(t^{\prime}+r\right) d r} d \xi(s) \leq\left(C_{29}+\epsilon\right)\left(1+|x|^{p}\right)
$$

for some arbitrary $\epsilon>0$. Using Lemma 1.8, we get $\mathbb{E} \xi(T-t) \leq \mathbb{E} \xi\left(T-t^{\prime}\right) \leq$ $C_{17}\left(1+|x|^{p}\right)$ and

$$
B \leq C_{35}\left|t-t^{\prime}\right|\left(1+|x|^{p}\right), \text { where } C_{35}=\left(c_{\max } T+1\right) L C_{17} .
$$


Now we estimate $A$ :

$$
\begin{aligned}
A \leq \mathbb{E} & \int_{0}^{T-t} \mid f\left(y_{x t}(t+s), t+s\right) e^{-\int_{0}^{s} \alpha(t+r) d r} \\
& -f\left(y_{x t}(t+s), t+s\right) e^{-\int_{0}^{s} \alpha\left(t^{\prime}+r\right) d r} \mid d s \\
+ & \mathbb{E} \int_{0}^{T-t} \mid f\left(y_{x t}(t+s), t+s\right) e^{-\int_{0}^{s} \alpha\left(t^{\prime}+r\right) d r} \\
& -f\left(y_{x t^{\prime}}\left(t^{\prime}+s\right), t^{\prime}+s\right) e^{-\int_{0}^{s} \alpha\left(t^{\prime}+r\right) d r} \mid d s \\
= & A_{1}+A_{2} .
\end{aligned}
$$

Using the inequality $\left|e^{x}-e^{y}\right| \leq|x-y|$ for $x, y \leq 0$ again, we get

$$
\begin{aligned}
A_{1} & \leq \mathbb{E} \int_{0}^{T-t} f\left(y_{x t}(t+s), t+s\right)\left(\int_{0}^{s}\left|\alpha(t+r)-\alpha\left(t^{\prime}+r\right)\right| d r\right) d s \\
& \leq T L\left|t-t^{\prime}\right| \mathbb{E} \int_{0}^{T-t} f\left(y_{x t}(t+s), t+s\right) d s .
\end{aligned}
$$

By virtue of (29) we can consider only those controls $v$ for which

$$
\mathbb{E} \int_{0}^{T-t^{\prime}} f\left(y_{x t^{\prime}}\left(t^{\prime}+s\right), t^{\prime}+s\right) e^{-\int_{0}^{s} \alpha\left(t^{\prime}+r\right) d r} d s \leq\left(C_{29}+\epsilon\right)\left(1+|x|^{p}\right)
$$

for some arbitrary $\epsilon>0$. Using (36) and Lemma 1.13, we get

$$
A_{1} \leq C_{38}\left|t-t^{\prime}\right|\left(1+|x|^{p}\right), \text { where } C_{38}=T L C_{27} .
$$

To estimate $A_{2}$ we use (7)-(8) and we have that $A_{2}$ is less than or equal to

$$
\begin{aligned}
& \mathbb{E} \int_{0}^{T-t} \mid f\left(y_{x t}(t+s), t+s\right)-f\left(y_{x t^{\prime}}\left(t^{\prime}+s\right), t+s\right) \\
& \quad+f\left(y_{x t^{\prime}}\left(t^{\prime}+s\right), t+s\right)-f\left(y_{x t^{\prime}}\left(t^{\prime}+s\right), t^{\prime}+s\right) \mid d s \\
& \leq \mathbb{E} \int_{0}^{T-t} C_{0}\left(1+f\left(y_{x t}(t+s), t+s\right)+f\left(y_{x t^{\prime}}\left(t^{\prime}+s\right), t+s\right)\right)^{1-1 / p} \\
& \quad \times\left|y_{x t^{\prime}}\left(t^{\prime}+s\right)-y_{x t}(t+s)\right| d s \\
& \quad+\mathbb{E} \int_{0}^{T-t} C_{0}\left(1+\left|y_{x t^{\prime}}\left(t^{\prime}+s\right)\right|^{p}\right)\left|t-t^{\prime}\right| d s=A_{3}+A_{4} .
\end{aligned}
$$

Using the Hölder's inequality and the Fubini's theorem, we get

$$
\begin{aligned}
A_{3} \leq C_{0} & \left\{\mathbb{E} \int_{0}^{T-t}\left(1+f\left(y_{x t}(t+s), t+s\right)+f\left(y_{x t^{\prime}}\left(t^{\prime}+s\right), t+s\right)\right) d s\right\}^{1-1 / p} \\
& \times\left\{\int_{0}^{T-t} \mathbb{E}\left|y_{x t^{\prime}}\left(t^{\prime}+s\right)-y_{x t}(t+s)\right|^{p} d s\right\}^{1 / p} .
\end{aligned}
$$


From this together with (37), Lemma 1.13, Lemma 1.10 and Lemma 1.11 we have

$$
A_{3} \leq C_{0}\left\{\left(T+C_{27}+C_{19}\right)\left(1+|x|^{p}\right)\right\}^{1-1 / p} \cdot\left\{T C_{20}\left|t-t^{\prime}\right|^{p}\left(1+|x|^{p}\right)\right\}^{1 / p} .
$$

Because $1+|x|^{p} \leq(1+|x|)^{p}$, we get

$$
A_{3} \leq C_{0}\left(T+C_{27}+C_{19}\right)^{1-1 / p}(1+|x|)^{p-1} \cdot\left(T C_{20}\right)^{1 / p}\left|t-t^{\prime}\right|(1+|x|) .
$$

Hence, from Lemma 1.2,

$$
A_{3} \leq C_{39}\left|t-t^{\prime}\right|\left(1+|x|^{p}\right),
$$

where $C_{39}=C_{0}\left(T+C_{27}+C_{19}\right)^{1-1 / p}\left(T C_{20}\right)^{1 / p} 2^{p-1}$. Furthermore, from Lemma 1.9 we get

$$
A_{4} \leq C_{40}\left|t-t^{\prime}\right|\left(1+|x|^{p}\right), \text { where } C_{40}=C_{0} C_{18} .
$$

In view of (34)-(35) and (38)-(40) we get for $t^{\prime} \leq t$,

$$
u(x, t)-u\left(x, t^{\prime}\right) \leq C_{41}\left|t-t^{\prime}\right|\left(1+|x|^{p}\right),
$$

where $C_{41}=C_{35}+C_{38}+C_{39}+C_{40}$.

To obtain a similar inequality for $t<t^{\prime}$ we proceed as follows. Let $\left(y_{x t}^{0}(s)\right)_{s \in[t, T]}$ be a solution of (1) with $v \equiv 0$. We can write the $i$-th coordinate of $y_{x t}^{0}(s)$ as follows

$$
y_{x t}^{0}(s)_{i}=x_{i}+\int_{t}^{s}\left(\sum_{j=1}^{n} a_{i j}(r) y_{x t}^{0}(r)_{j}+b_{i}(r)\right) d r+\sum_{j=1}^{n} \int_{t}^{s} \sigma_{i j}(r) d W_{r-t}^{j},
$$

$i=1, \ldots, n$, where subscripts denote the corresponding coordinates. Let $\left\{u_{m}(\cdot)\right\}_{n \in \mathbb{N}}$ be a sequence of mollifications of the function $u\left(\cdot, t^{\prime}\right)$ (see Def. 1.15). Applying the Itô's formula ([7], Th. 3.3.6), we get

$$
\begin{aligned}
& \mathbb{E} u_{m}\left(y_{x t}^{0}\left(t^{\prime}\right)\right) \\
& =u_{m}(x)+\mathbb{E} \sum_{i=1}^{n} \int_{t}^{t^{\prime}} \frac{\partial u_{m}\left(y_{x t}^{0}(s)\right)}{\partial x_{i}}\left(\sum_{j=1}^{n} a_{i j}(s) y_{x t}^{0}(s)_{j}+b_{i}(s)\right) d s \\
& \quad+\mathbb{E} \sum_{i=1}^{n} \int_{t}^{t^{\prime}} \frac{\partial u_{m}\left(y_{x t}^{0}(s)\right)}{\partial x_{i}} \sum_{j=1}^{n} \sigma_{i j}(s) d W_{s-t}^{j} \\
& \quad+\frac{1}{2} \mathbb{E} \sum_{i, j=1}^{n} \int_{t}^{t^{\prime}} \frac{\partial^{2} u_{m}\left(y_{x t}^{0}(s)\right)}{\partial x_{i} \partial x_{j}} d\left[y_{x t}^{0}(s)_{i}, y_{x t}^{0}(s)_{j}\right] \\
& =u_{m}(x)+\mathbb{A}+\mathbb{B}+\mathbb{C} .
\end{aligned}
$$

We need the following lemma. 
Lemma 2.3. We assume (29)-(31). Let $t^{\prime} \in[0, T]$ be fixed. Then there exist constants $C_{45}, C_{46}>0$ such that for all $x \in \mathbb{R}^{n}, m \in \mathbb{N}$ and $i, j \in\{1, \ldots, n\}$,

$$
\begin{gathered}
\lim _{m \rightarrow \infty} u_{m}(x)=u\left(x, t^{\prime}\right), \\
\left|\frac{\partial u_{m}(x)}{\partial x_{i}}\right| \leq C_{45}(1+|x|)^{p-1}, \\
0 \leq \frac{\partial^{2} u_{m}(x)}{\partial x_{i} \partial x_{j}} \leq C_{46}\left(1+|x|^{p}\right) .
\end{gathered}
$$

We estimate $\mathbb{A}$ as follows

$$
\begin{aligned}
\mathbb{A} & \leq \mathbb{E} \sum_{i=1}^{n} \int_{t}^{t^{\prime}}\left|\frac{\partial u_{m}\left(y_{x t}^{0}(s)\right)}{\partial x_{i}}\right| \cdot\left|\sum_{j=1}^{n} a_{i j}(s) y_{x t}^{0}(s)_{j}+b_{i}(s)\right| d s \\
& \leq \mathbb{E} \sum_{i=1}^{n} \int_{t}^{t^{\prime}}\left|\frac{\partial u_{m}\left(y_{x t}^{0}(s)\right)}{\partial x_{i}}\right|\left(n|| a(s)|| \cdot\left|y_{x t}^{0}(s)\right|+|b(s)|\right) d s .
\end{aligned}
$$

Using Lemma 2.3 and Lemma 1.2 , we see that $\mathbb{A}$ is not greater than

$$
\begin{gathered}
\sum_{i=1}^{n} \mathbb{E} \int_{t}^{t^{\prime}} C_{45}\left(1+\left|y_{x t}^{0}(s)\right|\right)^{p-1} n\left(a_{\max }+b_{\max }\right)\left(1+\left|y_{x t}^{0}(s)\right|\right) d s \\
\leq C_{47} \mathbb{E} \int_{t}^{t^{\prime}}\left(1+\left|y_{x t}^{0}(s)\right|^{p}\right) d s,
\end{gathered}
$$

where $C_{47}=n^{2} C_{45}\left(a_{\max }+b_{\max }\right) 2^{p-1}$.

Now we show that $\mathbb{B}=0$. Indeed,

$\mathbb{B}=\mathbb{E} \sum_{i, j=1}^{n} Z_{i j}\left(t^{\prime}\right)$, where $Z_{i j}(s)=\int_{t}^{s} \frac{\partial u_{m}\left(y_{x t}^{0}(r)\right)}{\partial x_{i}} \sigma_{i j}(r) d W_{r-t}^{j}$ for $s \in\left[t, t^{\prime}\right]$.

From properties of the Itô's integrals (see [7], Section 3.2) the process $\left(Z_{i j}(s)\right)_{s \in\left[t, t^{\prime}\right]}$ is a martingale provided that

$$
\mathbb{E} \int_{t}^{t^{\prime}}\left(\frac{\partial u_{m}\left(y_{x t}^{0}(s)\right)}{\partial x_{i}} \sigma_{i j}(s)\right)^{2} d s \leq \infty .
$$

Using Lemma 2.3 and Lemma 1.2, we have

$$
\begin{aligned}
& \mathbb{E} \int_{t}^{t^{\prime}}\left(\frac{\partial u_{m}\left(y_{x t}^{0}(s)\right)}{\partial x_{i}} \sigma_{i j}(s)\right)^{2} d s \leq \mathbb{E} \int_{t}^{t^{\prime}} C_{45}^{2}\left(1+\left|y_{x t}^{0}(s)\right|\right)^{2 p-2} \sigma_{\max }^{2} d s \\
& \leq C_{45}^{2} \sigma_{\max }^{2} \mathbb{E} \int_{t}^{t^{\prime}}\left(1+\left|y_{x t}^{0}(s)\right|\right)^{2 p} d s \\
& \leq C_{45}^{2} \sigma_{\max }^{2} 2^{2 p-1} \mathbb{E} \int_{t}^{t^{\prime}}\left(1+\left|y_{x t}^{0}(s)\right|^{2 p}\right) d s .
\end{aligned}
$$


Using the Fubini's theorem and Lemma 1.3, we get

$$
\begin{aligned}
& \mathbb{E} \int_{t}^{t^{\prime}}\left(\frac{\partial u_{m}\left(y_{x t}^{0}(s)\right)}{\partial x_{i}} \sigma_{i j}(s)\right)^{2} d s \\
& \quad \leq C_{45}^{2} \sigma_{\max }^{2} 2^{2 p-1} \int_{t}^{t^{\prime}}\left(1+C_{11} e^{C_{11} T}(1+|x|)^{2 p}\right) d s<\infty .
\end{aligned}
$$

Hence $Z_{i j}$ is a martingale and $\mathbb{E} Z_{i j}\left(t^{\prime}\right)=\mathbb{E} Z_{i j}(t)=0$. So

$$
\mathbb{B}=\mathbb{E} \sum_{i, j=1}^{n} Z_{i j}\left(t^{\prime}\right)=0 .
$$

Now, using the conventional "multiplication rules" (see [7], p. 154), we know that

$$
d s d s=0, \quad d s d W_{s}^{i}=0, \quad d W_{s}^{i} d W_{s}^{i}=d s, \quad d W_{s}^{i} d W_{s}^{j}=0 \quad \text { for } i \neq j .
$$

So in view of $(42)$ we can write

$$
d\left[y_{x t}^{0}(s)_{i}, y_{x t}^{0}(s)_{j}\right]=\sum_{k=1}^{n} \sigma_{i k}(s) d W_{s-t}^{k} \cdot \sum_{l=1}^{n} \sigma_{j l}(s) d W_{s-t}^{l}=\sum_{k=1}^{n} \sigma_{i k}(s) \sigma_{j k}(s) d s .
$$

From Lemma 2.3 we have

$$
\begin{aligned}
\mathbb{C} & =\frac{1}{2} \sum_{i, j=1}^{n} \mathbb{E} \int_{t}^{t^{\prime}} \frac{\partial^{2} u_{m}\left(y_{x t}^{0}(s)\right)}{\partial x_{i} \partial x_{j}} \sum_{k=1}^{n} \sigma_{i k}(s) \sigma_{j k}(s) d s \\
& \leq \frac{1}{2} \sum_{i, j=1}^{n} \mathbb{E} \int_{t}^{t^{\prime}} C_{46}\left(1+\left|y_{x t}^{0}(s)\right|^{p}\right) n \sigma_{\max }^{2} d s \\
& =C_{49} \mathbb{E} \int_{t}^{t^{\prime}}\left(1+\left|y_{x t}^{0}(s)\right|^{p}\right) d s, \text { where } C_{49}=\frac{1}{2} C_{46} \sigma_{\max }^{2} n^{3} .
\end{aligned}
$$

In summary, in view of (43) and (47)-(49)

$$
\mathbb{E} u_{m}\left(y_{x t}^{0}\left(t^{\prime}\right)\right) \leq u_{m}(x)+\left(C_{47}+C_{49}\right) \mathbb{E} \int_{t}^{t^{\prime}}\left(1+\left|y_{x t}^{0}(s)\right|^{p}\right) d s .
$$

Taking the limit as $n \rightarrow \infty$ and using the Fatou's lemma, we get

$$
\mathbb{E} u\left(y_{x t}^{0}\left(t^{\prime}\right), t^{\prime}\right) \leq u\left(x, t^{\prime}\right)+C_{50} \mathbb{E} \int_{t}^{t^{\prime}}\left(1+\left|y_{x t}^{0}(s)\right|^{p}\right) d s,
$$

$C_{50}=C_{47}+C_{49}$. Furthermore, from Lemma 1.2 and Lemma 1.3 we have for each $s \in[t, T]$

$$
\mathbb{E}\left(1+\left|y_{x t}^{0}(s)\right|^{p}\right) \leq C_{51}\left(1+|x|^{p}\right), \quad C_{51}=C_{11} e^{C_{11} T} 2^{p-1}+1 .
$$


Next, from (5), (6), (50), (51) and the Fubini's theorem we conclude

$$
\begin{aligned}
u(x, t) & \leq \mathbb{E}\left\{\int_{t}^{t^{\prime}} f\left(y_{x t}^{0}(s), s\right) e^{-\int_{t}^{s} \alpha(r) d r} d s+u\left(y_{x t}^{0}\left(t^{\prime}\right), t^{\prime}\right) e^{-\int_{t}^{t^{\prime}} \alpha(r) d r}\right\} \\
& \leq C_{0} \int_{t}^{t^{\prime}} \mathbb{E}\left(1+\left|y_{x t}^{0}(s)\right|^{p}\right) d s+\mathbb{E} u\left(y_{x t}^{0}\left(t^{\prime}\right), t^{\prime}\right) \\
& \leq\left(C_{0} C_{51}+C_{50} C_{51}\right)\left(\left|t-t^{\prime}\right|\left(1+|x|^{p}\right)\right)+u\left(x, t^{\prime}\right) .
\end{aligned}
$$

Hence, for $t<t^{\prime}$

$$
u(x, t)-u\left(x, t^{\prime}\right) \leq C_{52}\left|t-t^{\prime}\right|\left(1+|x|^{p}\right), \quad C_{52}=C_{51}\left(C_{0}+C_{50}\right) .
$$

It is clear that (41) and (52) imply (33).

Now we give the proof of Lemma 2.3.

Proof: Proof of (44). The continuity of $u\left(\cdot, t^{\prime}\right)$ is a consequence of (30). So in view of Lemma 1.16 we conclude that $\lim _{m \rightarrow \infty} u_{m}(x)=u\left(x, t^{\prime}\right)$.

Proof of (45). Let $x \in \mathbb{R}^{n}, 0 \leq\left|x^{\prime}\right|<1$. From Definitions 1.14, 1.15 and (30) we get

$$
\begin{aligned}
& \left|u_{m}(x)-u_{m}\left(x+x^{\prime}\right)\right|=\left|\int_{B\left(0, \frac{1}{m}\right)} \eta_{m}(y)\left(u\left(x-y, t^{\prime}\right)-u\left(x+x^{\prime}-y, t^{\prime}\right)\right) d y\right| \\
& \quad \leq \int_{B\left(0, \frac{1}{m}\right)} m^{n} \cdot \eta(m y)\left|u\left(x-y, t^{\prime}\right)-u\left(x+x^{\prime}-y, t^{\prime}\right)\right| d y \\
& \quad \leq C_{28} C_{30}\left|x^{\prime}\right| m^{n} \int_{B\left(0, \frac{1}{m}\right)}\left(1+|x-y|^{p-1}+\left|x+x^{\prime}-y\right|^{p-1}\right) d y .
\end{aligned}
$$

Because $\left|x^{\prime}\right|<1$ and $|y| \leq \frac{1}{m} \leq 1$, we have

$$
\begin{aligned}
1+|x-y|^{p-1}+\left|x+x^{\prime}-y\right|^{p-1} & \leq 1+(1+|x|)^{p-1}+(2+|x|)^{p-1} \\
& \leq\left(2+2^{p-1}\right)(1+|x|)^{p-1} .
\end{aligned}
$$

Furthermore (see [4], p. 615),

$$
\int_{B\left(0, \frac{1}{m}\right)} d y=\frac{\Pi^{n / 2}}{\Gamma\left(\frac{n}{2}+1\right)} \cdot \frac{1}{m^{n}}, \text { where } \Gamma(t)=\int_{0}^{\infty} s^{t-1} e^{-s} d s, \text { for } t>0 .
$$

In summary,

$$
\frac{\left|u_{m}(x)-u_{m}\left(x+x^{\prime}\right)\right|}{\left|x^{\prime}\right|} \leq C_{28} C_{30} \frac{\Pi^{n / 2}}{\Gamma\left(\frac{n}{2}+1\right)}\left(2+2^{p-1}\right)(1+|x|)^{p-1} .
$$

Taking the limit as $\left|x^{\prime}\right| \rightarrow 0$ on both sides, we conclude (45). 
Proof of (46). Let $x^{\prime} \in \mathbb{R}^{n}$ and $\lambda \in(0,1)$. We have

$$
\begin{aligned}
u_{m}\left(x+\lambda x^{\prime}\right) & -2 u_{m}(x)+u_{m}\left(x-\lambda x^{\prime}\right) \\
& =\int_{B\left(0, \frac{1}{m}\right)} \eta_{m}(y)\left(u\left(x+\lambda x^{\prime}, t^{\prime}\right)-2 u\left(x, t^{\prime}\right)+u\left(x-\lambda x^{\prime}, t^{\prime}\right)\right) d y .
\end{aligned}
$$

From (31) and nonnegativity of $\eta_{m}$ we conclude that $\frac{\partial^{2} u_{m}(x)}{\partial x_{i} \partial x_{j}} \geq 0$. On the other hand, using (31) and mimicking the proof of (45), we see that

$$
\begin{aligned}
u_{m}\left(x+\lambda x^{\prime}\right) & -2 u_{m}(x)+u_{m}\left(x-\lambda x^{\prime}\right) \\
& \leq \int_{B\left(0, \frac{1}{m}\right)} m^{n} \cdot \eta(m y) C_{31} \lambda^{2}(1+|x|)^{(p-2)^{+}} d y \\
& \leq \lambda^{2} C_{28} C_{31} \frac{\Pi^{n / 2}}{\Gamma\left(\frac{n}{2}+1\right)}(1+|x|)^{(p-2)^{+}} .
\end{aligned}
$$

For $p \in(1,2],(1+|x|)^{(p-2)^{+}}=1 \leq\left(1+|x|^{p}\right) \leq 2^{p-1}\left(1+|x|^{p}\right)$. For $p>2$, in view of Lemma $1.2,(1+|x|)^{(p-2)^{+}}=(1+|x|)^{p-2} \leq(1+|x|)^{p} \leq 2^{p-1}\left(1+|x|^{p}\right)$. Thus, for all $p>1$ we have

$$
\frac{u_{m}\left(x+\lambda x^{\prime}\right)-2 u_{m}(x)+u_{m}\left(x-\lambda x^{\prime}\right)}{\lambda^{2}} \leq C_{28} C_{31} \frac{\Pi^{n / 2}}{\Gamma\left(\frac{n}{2}+1\right)} 2^{p-1}\left(1+|x|^{p}\right) .
$$

Taking the limit as $\lambda \rightarrow 0$, we can conclude (46).

Remark 2.4. Theorems 2.1 and 2.2 are true for functions $u_{\epsilon}$ (see (4)) instead of $u$. Indeed, in view of the proofs we see that the constants $C_{29}, C_{30}, C_{31}, C_{33}$ do not depend on $\epsilon$.

Remark 2.5. It follows from (44)-(46) that for every $t^{\prime} \in[0, T] D u_{m}\left(\cdot ; t^{\prime}\right)$ converges to $D u\left(\cdot, t^{\prime}\right)$ the distributional gradient of $u$ with respect to $x$ almost uniformly as $m \rightarrow \infty$ (see the proof of Theorem 3.5, to follow, for a similar argument, with $u_{m}$ replaced by $u_{\epsilon_{m}}$ ). This implies differentiability of $u$ with respect to $x$ in the classical sense (see, e.g., Theorem 7.17 in [13]), so $D u$ is the classical gradient of $u$ with respect to $x$ at any point $\left(x, t^{\prime}\right) \in$ $\mathbb{R}^{n} \times[0, T]$. Moreover, by (46) $D u_{m}$ are locally Lipschitz in $x$ uniformly in $m$, so $D u$ is also locally Lipschitz in $x$. Thus Theorems 2.1, 2.2 and their proofs imply that the value function $u(x, t)$ has generalized derivatives of the first order with respect to $t$ and of the second order with respect to $x$. These generalized derivatives belongs to the space $L_{l o c}^{\infty}\left(\mathbb{R}^{n} \times[0, T]\right)$ of all functions essentially bounded on every open bounded subset of the domain.

Proposition 2.6. For all $x \in \mathbb{R}^{n}$ and $t \in[0, T]$ we have $u(x, t) \leq\left(c_{\max }+\right.$ $\left.C_{29}\right)(1+|x|)$. 
Proof. Let $x^{\prime} \in \mathbb{R}^{n}$ be arbitrary. Consider controls for which $\lim _{s \rightarrow 0+} v_{s}=$ $x$. In view of (2) and (3) we have

$$
\begin{aligned}
u\left(x^{\prime}, t\right) & =\inf \left\{J_{x^{\prime} t}(v): v \in \mathcal{V}\right\} \leq c(t)|x|+\inf \left\{J_{x+x^{\prime}, t}(v): v \in \mathcal{V}\right\} \\
& =c(t)|x|+u\left(x+x^{\prime}, t\right) .
\end{aligned}
$$

So $u\left(x^{\prime}, t\right)-u\left(x+x^{\prime}, t\right) \leq c(t)|x|$. Similarly $u\left(x+x^{\prime}, t\right)-u\left(x^{\prime}, t\right) \leq c(t)|x|$, so

$$
\left|u\left(x+x^{\prime}, t\right)-u\left(x^{\prime}, t\right)\right| \leq c(t)|x| .
$$

Taking $x^{\prime}=0$, we get $|u(x, t)-u(0, t)| \leq c(t)|x|$. From (29) we see that $u(0, t) \leq C_{29}$ so $u(x, t) \leq c(t)|x|+u(0, t) \leq c_{\max }|x|+C_{29} \leq\left(c_{\max }+C_{29}\right)(1+$ $|x|)$.

Remark 2.7. The proof of Proposition 2.6 is not valid for $u_{\epsilon}$ instead of $u$, because if a control $v \in \mathcal{V}_{\epsilon}$, then it is continuous, so the condition $\lim _{s \rightarrow 0+} v_{s}=x$ is invalid for $x \neq 0$.

Remark 2.8. The value function $u(x, t)$ satisfies $|D u(x, t)| \leq c(t)$ for all $(x, t) \in \mathbb{R}^{n} \times[0, T]$. Indeed, the gradient exists for all $(x, t) \in \mathbb{R}^{n} \times[0, T]$ in view of Remark 2.5. From (53) we see that the first derivative of $u(x, t)$ with respect to $x$ in any direction is bounded by $c(t)$. Hence, the norm of the gradient $D u(x, t)$ is bounded by $c(t)$, too.

3. Dynamic Programming Principle and HJB equation. To consider the DPP and the HJB equation for our problem we will first prove the pointwise convergence of $u_{\epsilon}$ to $u$ if $\epsilon \rightarrow 0^{+}$. For this purpose we need an integral form of the Gronwall's inequality with locally finite measures.

Lemma 3.1 (see [18]). Let $\mu$ be a locally finite measure on the Borel $\sigma$ algebra of $[t, T]$, where $0 \leq t \leq T$. We consider a measurable function $\phi$ defined on $[t, T]$ such that $\int_{t}^{T}|\phi(r)| \mu(d r)<\infty$. We assume that there exists a Borel function $\psi \geq 0$ on $[t, T]$ such that for all $s \in[t, T]$,

$$
\phi(s) \leq \psi(s)+\int_{[t, s)} \phi(r) \mu(d r) .
$$

Then for all $s \in[t, T]$,

$$
\phi(s) \leq \psi(s)+\int_{[t, s)} \psi(r) e^{\mu([r, s))} \mu(d r) .
$$

Theorem 3.2. For all $(x, t) \in \mathbb{R}^{n} \times[0, T]$ we have $\lim _{\epsilon \rightarrow 0^{+}} u_{\epsilon}(x, t)=u(x, t)$.

Proof. Fix $x \in \mathbb{R}^{n}$ and $t \in[0, T]$. Consider an arbitrary $v \in \mathcal{V}$ such that $J_{x t}(v)<\infty$.

Step 1. We show first that $v \in L^{p}\left(\Omega \times[0, T-t], P \otimes \mu_{L e b}\right)$, where $\mu_{L e b}$ denotes the Lebesgue's measure. Since $J_{x t}(v)<\infty$, we have

$$
\mathbb{E} \int_{t}^{T} f\left(y_{x t}(s), s\right) d s<\infty
$$


and from (6) we get

$$
\mathbb{E} \int_{t}^{T}\left|y_{x t}(s)\right|^{p} d s<\infty .
$$

From (1) we can write for $s \in[t, T]$

$$
v(s-t)=y_{x t}(s)-x-\int_{t}^{s} b(r) d r-\int_{t}^{s} \sigma(r) d W_{r-t}-\int_{t}^{s} a(r) y_{x t}(r) d r .
$$

Using (54) and properties of the normal distribution, we know that each term from the line above, maybe except for the last one, belongs to the space $L^{p}(\Omega \times[0, T-t])$. But the last term belongs to this space, too. Indeed,

$$
\mathbb{E} \int_{t}^{T}\left|\int_{t}^{s} a(r) y_{x t}(r) d r\right|^{p} d s \leq a_{\max }^{p} \mathbb{E} \int_{t}^{T}\left(\int_{t}^{T}\left|y_{x t}(r)\right| d r\right)^{p} d s .
$$

Using the Hölder's inequality and (54), we can estimate the last expression above by

$$
\begin{aligned}
a_{\max }^{p} \mathbb{E} \int_{t}^{T}\left(\int_{t}^{T}\left|y_{x t}(r)\right|^{p} d r \cdot|T-t|^{p / q}\right) d s \\
\leq a_{\max }^{p} T^{1+p / q} \mathbb{E} \int_{t}^{T}\left|y_{x t}(r)\right|^{p} d r<\infty,
\end{aligned}
$$

where $\frac{1}{p}+\frac{1}{q}=1$. Hence, from (55) we see that $v \in L^{p}(\Omega \times[0, T-t])$.

Step 2. Now we define a sequence of bounded controls $\left\{v_{R}, R>0\right\}$ such that $v_{R}$ is convergent to $v$ in the space $L^{p}(\Omega \times[0, T-t])$ and the total variation of $v_{R}$ is pointwise convergent to the total variation of $v$ from below. Let

$$
v_{R}(s)= \begin{cases}v(s), & |v(s)| \leq R \\ \frac{v(s)}{|v(s)|} \cdot R, & |v(s)|>R\end{cases}
$$

We see that for all $s \in[0, T-t] \lim _{R \rightarrow \infty} v_{R}(s)=v(s)$ and $\left|v_{R}(s)\right| \leq|v(s)|$. Hence, from Lemma 1.2 and Step 1,

$$
\mathbb{E} \int_{0}^{T-t}\left|v(s)-v_{R}(s)\right|^{p} d s \leq 2^{p} \mathbb{E} \int_{0}^{T-t}|v(s)|^{p} d s<\infty
$$

and using the Lebesgue's dominated convergence theorem, we get

$$
\lim _{R \rightarrow \infty} \mathbb{E} \int_{0}^{T-t}\left|v(s)-v_{R}(s)\right|^{p} d s=\mathbb{E} \int_{0}^{T-t} \lim _{R \rightarrow \infty}\left|v(s)-v_{R}(s)\right|^{p} d s=0 .
$$

The convergence in $L^{p}$ is proved. Moreover, if $\xi(s), \xi_{R}(s)$ denote the total variations on the interval $[0, s]$ of the functions $v, v_{R}$ respectively, then for all $s \in[0, T-t]$,

$$
\xi_{R}(s) \leq \xi(s) \text { and } \lim _{R \rightarrow \infty} \xi_{R}(s)=\xi(s)
$$


Step 3. Let $y_{x t}^{v}, y_{x t}^{v_{R}}$ denote the state processes (see (1)) corresponding to the controls $v, v_{R}$ respectively. We want to show that $\left\{y_{x t}^{v_{R}}\right\}$ is convergent to $y_{x t}^{v}$ in the space $L^{p}(\Omega \times[t, T])$. First we observe that for $s \in[t, T]$,

$$
y_{x t}^{v}(s)-y_{x t}^{v_{R}}(s)=\int_{t}^{s} a(r)\left(y_{x t}^{v}(r)-y_{x t}^{v_{R}}(r)\right) d r+v(s-t)-v_{R}(s-t) .
$$

Denoting $z_{R}(s)=y_{x t}^{v}(s)-y_{x t}^{v_{R}}(s)$ and $u_{R}(s)=v(s-t)-v_{R}(s-t)$, we can rewrite the last equality in the form $z_{R}(s)=\int_{t}^{s} a(r) z_{R}(r) d r+u_{R}(s)$. Hence $\left|z_{R}(s)\right| \leq \int_{t}^{s}\left|z_{R}(r)\right| a_{\max } d r+\left|u_{R}(s)\right|$. Using Lemma 3.1 with $\phi=\left|z_{R}\right|$, $\psi=\left|u_{R}\right|$ and $\mu=a_{\max } \cdot \mu_{L e b}$, we get

$$
\begin{aligned}
\left|z_{R}(s)\right| & \leq\left|u_{R}(s)\right|+\int_{t}^{s}\left|u_{R}(r)\right| e^{a_{\max }(s-r)} d r \\
& \leq\left|u_{R}(s)\right|+C_{57} \int_{t}^{s}\left|u_{R}(r)\right| d r,
\end{aligned}
$$

where $C_{57}=e^{a_{\max } T}$. So from Lemma 1.2 and the Hölder's inequality

$$
\begin{aligned}
\left|z_{R}(s)\right|^{p} & \leq 2^{p-1}\left\{\left|u_{R}(s)\right|^{p}+C_{57}^{p}\left(\int_{t}^{s}\left|u_{R}(r)\right| d r\right)^{p}\right\} \\
& \leq 2^{p-1}\left\{\left|u_{R}(s)\right|^{p}+C_{57}^{p}(s-t)^{p / q} \int_{t}^{s}\left|u_{R}(r)\right|^{p} d r\right\} \\
& \leq C_{58}\left\{\left|u_{R}(s)\right|^{p}+\int_{t}^{T}\left|u_{R}(r)\right|^{p} d r\right\}
\end{aligned}
$$

where $\frac{1}{p}+\frac{1}{q}=1$ and $C_{58}=2^{p-1}\left(1+C_{57}^{p} T^{p / q}\right)$. Finally, in view of Step 2 we have

$$
\begin{gathered}
\lim _{R \rightarrow \infty} \mathbb{E} \int_{t}^{T}\left|z_{R}(s)\right|^{p} d s \leq \lim _{R \rightarrow \infty} C_{58} \mathbb{E} \int_{t}^{T}\left\{\left|u_{R}(s)\right|^{p}+\int_{t}^{T}\left|u_{R}(r)\right|^{p} d r\right\} d s \\
\leq \lim _{R \rightarrow \infty}\left\{C_{58} \mathbb{E} \int_{t}^{T}\left|u_{R}(s)\right|^{p} d s+C_{58} T \mathbb{E} \int_{t}^{T}\left|u_{R}(r)\right|^{p} d r\right\}=0 .
\end{gathered}
$$

Step 4. The next step is to show that $J_{x t}\left(v_{R}\right) \rightarrow J_{x t}(v)$ if $R \rightarrow \infty$. Indeed,

$$
\begin{aligned}
&\left|J_{x t}(v)-J_{x t}\left(v_{R}\right)\right| \leq\left|\mathbb{E} \int_{t}^{T}\left(f\left(y_{x t}^{v}(s), s\right)-f\left(y_{x t}^{v_{R}}(s), s\right)\right) e^{-\int_{t}^{s} \alpha(r) d r} d s\right| \\
&+\left|\mathbb{E} \int_{t}^{T} c(s) e^{-\int_{t}^{s} \alpha(r) d r} d\left(\xi-\xi_{R}\right)(s-t)\right|=A_{R}+B_{R} .
\end{aligned}
$$


In view of $(56)$,

$$
B_{R} \leq c_{\max }\left|\mathbb{E} \int_{t}^{T} d\left(\xi-\xi_{R}\right)(s-t)\right|=c_{\max } \mathbb{E}\left(\xi(T-t)-\xi_{R}(T-t)\right) .
$$

Using (56) again and the assumption that $J_{x t}(v)<\infty$, we see that $\mathbb{E}(\xi(T-t)$ $\left.-\xi_{R}(T-t)\right) \leq \mathbb{E} \xi(T-t)<\infty$. Hence, from the Lebesgue's dominated convergence theorem we get

$$
\begin{aligned}
\lim _{R \rightarrow \infty} B_{R} & \leq \lim _{R \rightarrow \infty} c_{\max } \mathbb{E}\left(\xi(T-t)-\xi_{R}(T-t)\right) \\
& =c_{\max } \mathbb{E} \lim _{R \rightarrow \infty}\left(\xi(T-t)-\xi_{R}(T-t)\right)=0 .
\end{aligned}
$$

Using (7) and the Hölder's inequality, we have

$$
\begin{gathered}
A_{R} \leq \mathbb{E} \int_{t}^{T}\left|f\left(y_{x t}^{v}(s), s\right)-f\left(y_{x t}^{v_{R}}(s), s\right)\right| d s \\
\leq \mathbb{E} \int_{t}^{T}\left(1+f\left(y_{x t}^{v}(s), s\right)+f\left(y_{x t}^{v_{R}}(s), s\right)\right)^{1-1 / p}\left|y_{x t}^{v}(s)-y_{x t}^{v_{R}}(s)\right| d s \\
\leq\left\{\mathbb{E} \int_{t}^{T}\left(1+f\left(y_{x t}^{v}(s), s\right)+f\left(y_{x t}^{v_{R}}(s), s\right)\right) d s\right\}^{1-1 / p}\left\{\mathbb{E} \int_{t}^{T}\left|y_{x t}^{v}(s)-y_{x t}^{v_{R}}(s)\right|^{p}\right\}^{1 / p} .
\end{gathered}
$$

In view of Step 3, the second factor in the last expression goes to 0 if $R \rightarrow \infty$. We must show that the first factor is bounded. Indeed, from (6) and Lemma 1.2 we can write

$$
\begin{aligned}
& \mathbb{E} \int_{t}^{T}\left(1+f\left(y_{x t}^{v}(s), s\right)+f\left(y_{x t}^{v_{R}}(s), s\right)\right) d s \\
& \quad \leq\left(1+C_{0}\right) \mathbb{E} \int_{t}^{T}\left(2+\left|y_{x t}^{v}(s)\right|^{p}+\left|y_{x t}^{v_{R}}(s)\right|^{p}\right) d s \\
& \quad \leq\left(1+C_{0}\right) \mathbb{E} \int_{t}^{T}\left(2+\left|y_{x t}^{v}(s)\right|^{p}+2^{p-1}\left|y_{x t}^{v}(s)\right|^{p}+2^{p-1}\left|y_{x t}^{v}(s)-y_{x t}^{v_{R}}(s)\right|^{p}\right) d s .
\end{aligned}
$$

Using (54) and Step 3 again, we conclude that the last expression is bounded uniformly in $R$. Hence $\lim _{R \rightarrow \infty} A_{R}=0$.

Summarizing Steps 1-4, we know that $J_{x t}\left(v_{R}\right)$ goes to $J_{x t}(v)$ if $R \rightarrow \infty$, so we can consider only bounded controls.

Step 5. Consider $v \in \mathcal{V}$ such that $\|v\|_{\infty}<R$ for some $R>0$. We will construct a sequence of controls $\left\{v_{n}, n \in \mathbb{N}\right\}$ convergent to $v$ in $L^{p}(\Omega \times$ $[0, T-t])$ and such that $v_{n} \in V_{1 /(2 n R)}$ for all $n$. Besides we shall prove that the variation of $v_{n}$ is pointwise convergent to the variation of $v$ from below. Let $v_{n}(s)=n \int_{(s-1 / n) \vee 0}^{s} v(r) d r, s \in[0, T-t]$. We observe that $v_{n}$ is a progressively measurable continuous random process such that $\left\|v_{n}\right\|_{\infty} \leq R$, so $v_{n} \in L^{p}(\Omega \times[0, T-t])$. From left-continuity of $v$ we know that

$$
\forall_{\omega \in \Omega} \forall_{s \in[0, T-t]} \quad \lim _{n \rightarrow \infty} v_{n}(s)=v(s) .
$$


Using the Lebesgue's dominated convergence theorem, we get

$$
\lim _{n \rightarrow \infty} \mathbb{E} \int_{0}^{T-t}\left|v(s)-v_{n}(s)\right|^{p} d s=\mathbb{E} \int_{0}^{T-t} \lim _{n \rightarrow \infty}\left|v(s)-v_{n}(s)\right|^{p} d s=0,
$$

so $L^{p}$-convergence is proved.

Now we want to check that $v_{n} \in V_{1 /(2 n R)}$. Indeed,

$\left|\frac{d}{d s} v_{n}(s)\right|=\left|\frac{d}{d s}\left(n \int_{(s-1 / n) \vee 0}^{s} v(r) d r\right)\right|=n|v(s)-v((s-1 / n) \vee 0)| \leq 2 n R$.

Let $\xi_{n}(s), \xi(s)$ denote the variations on the interval $[0, s]$ of the functions $v_{n}, v$ respectively. For convenience, we define $v(r) \equiv 0$ for $r<0$. Then $v_{n}(s)=n \int_{s-1 / n}^{s} v(r) d r, s \in[0, T-t]$. Fix $\omega \in \Omega, s \in(0, T-t]$. Let $\Pi=\left\{s_{0}, s_{1}, \ldots, s_{k}\right\}$ be a partition of the interval $[0, s]$, where $0=s_{0}<s_{1}<$ $\cdots<s_{k}=s$. Then

$$
\begin{aligned}
& \sum_{i=1}^{k}\left|v_{n}\left(s_{i}\right)-v_{n}\left(s_{i-1}\right)\right|=n \sum_{i=1}^{k}\left|\int_{s_{i}-1 / n}^{s_{i}} v(r) d r-\int_{s_{i-1}-1 / n}^{s_{i-1}} v(r) d r\right| \\
& \quad=n \sum_{i=1}^{k}\left|\int_{0}^{1 / n}\left(v\left(s_{i}+r-1 / n\right)-v\left(s_{i-1}+r-1 / n\right)\right) d r\right| \\
& \quad \leq n \int_{0}^{1 / n} \sum_{i=1}^{k}\left|v\left(s_{i}+r-1 / n\right)-v\left(s_{i-1}+r-1 / n\right)\right| d r \\
& \leq n \int_{0}^{1 / n} \xi(s) d r=\xi(s) .
\end{aligned}
$$

Letting $\|\Pi\| \rightarrow 0$, we get

$$
\xi_{n}(s) \leq \xi(s)
$$

On the other hand, from (59) we see that

$$
\begin{aligned}
\sum_{i=1}^{k}\left|v\left(s_{i}\right)-v\left(s_{i-1}\right)\right| & =\sum_{i=1}^{k}\left|\lim _{n \rightarrow \infty} v_{n}\left(s_{i}\right)-\lim _{n \rightarrow \infty} v_{n}\left(s_{i-1}\right)\right| \\
& =\lim _{n \rightarrow \infty} \sum_{i=1}^{k}\left|v_{n}\left(s_{i}\right)-v_{n}\left(s_{i-1}\right)\right| \leq \liminf _{n \rightarrow \infty} \xi_{n}(s) .
\end{aligned}
$$

Letting $\|\Pi\| \rightarrow 0$ and using (60), we have

$$
\xi(s) \leq \liminf _{n \rightarrow \infty} \xi_{n}(s) \leq \limsup _{n \rightarrow \infty} \xi_{n}(s) \leq \xi(s) \quad \Leftrightarrow \quad \lim _{n \rightarrow \infty} \xi_{n}(s)=\xi(s) .
$$

Step 6. In view of Step 5 we can mimic Steps 3 and 4 to conclude that $J_{x t}\left(v_{n}\right) \rightarrow J_{x t}(v)$ if $n \rightarrow \infty$, where $\|v\|_{\infty}<R$ for some $R>0$. From this 
and Step 4, remembering that $v_{n} \in \mathcal{V}_{1 /(2 n R)}$, we can write

$$
\inf _{v \in \mathcal{V}} J_{x t}(v)=\inf _{v \in \bigcup_{\epsilon>0} \mathcal{V}_{\epsilon}} J_{x t}(v)
$$

and $\lim _{\epsilon \rightarrow 0^{+}} u_{\epsilon}(x, t)=u(x, t)$.

Theorem 3.3 (Bellman's dynamic programming principle). Let $x \in \mathbb{R}^{n}$, $t \in[0, T]$ and let $y_{x t}^{v}$ denote the state process corresponding to a control $v \in \mathcal{V}$. Let $\tau \in[0, T-t]$ be a Markov time with respect to $\left\{\mathcal{F}_{t}\right\}$. Then

$$
\begin{aligned}
u(x, t)=\inf _{v \in \mathcal{V}} \mathbb{E}\{ & \int_{t}^{t+\tau} f\left(y_{x t}^{v}(s), s\right) e^{-\int_{t}^{s} \alpha(r) d r} d s \\
& \left.+\int_{t}^{t+\tau} c(s) e^{-\int_{t}^{s} \alpha(r) d r} d \xi(s-t)+u\left(y_{x t}^{v}(t+\tau), t+\tau\right)\right\} .
\end{aligned}
$$

Proof. For convenience let us denote

$J_{x t}(v, \tau)=\mathbb{E}\left\{\int_{t}^{t+\tau} f\left(y_{x t}^{v}(s), s\right) e^{-\int_{t}^{s} \alpha(r) d r} d s+\int_{t}^{t+\tau} c(s) e^{-\int_{t}^{s} \alpha(r) d r} d \xi(s-t)\right\}$.

It is known that DPP holds for regular stochastic control problems (see, e.g., [10], Th. 3.1.6). Hence we have for each $\epsilon>0$,

$$
u_{\epsilon}(x, t)=\inf _{v \in \mathcal{V}_{\epsilon}}\left\{J_{x t}(v, \tau)+\mathbb{E} u_{\epsilon}\left(y_{x t}^{v}(t+\tau), t+\tau\right)\right\} .
$$

Considering any $\tilde{v} \in \mathcal{V}_{\epsilon}$, we have

$$
u_{\epsilon}(x, t) \leq J_{x t}(\tilde{v}, \tau)+\mathbb{E} u_{\epsilon}\left(y_{x t}^{\tilde{v}}(t+\tau), t+\tau\right) .
$$

If $\epsilon \rightarrow 0^{+}$, from Theorem 3.2 and the Lebesgue's dominated convergence theorem we get

$$
u(x, t) \leq J_{x t}(\tilde{v}, \tau)+\mathbb{E} u\left(y_{x t}^{\tilde{v}}(t+\tau), t+\tau\right) .
$$

Because $\epsilon>0$ and $\tilde{v} \in \mathcal{V}_{\epsilon}$ are arbitrary we can conclude that

$$
u(x, t) \leq \inf _{v \in \bigcup_{\epsilon>0} \mathcal{V}_{\epsilon}}\left\{J_{x t}(v, \tau)+\mathbb{E} u\left(y_{x t}^{v}(t+\tau), t+\tau\right)\right\} .
$$

On the other hand, from (62)

$$
u_{\epsilon}(x, t) \geq \inf _{v \in \bigcup_{\epsilon>0} \mathcal{V}_{\epsilon}}\left\{J_{x t}(v, \tau)+\mathbb{E} u\left(y_{x t}^{v}(t+\tau), t+\tau\right)\right\} .
$$

Letting $\epsilon \rightarrow 0^{+}$, we get

$$
u(x, t) \geq \inf _{v \in \bigcup_{\epsilon>0}}\left\{\mathcal{V}_{\epsilon}\left\{J_{x t}(v, \tau)+\mathbb{E} u\left(y_{x t}^{v}(t+\tau), t+\tau\right)\right\} .\right.
$$

The inequalities (63), (64) and an argument similar to the proof of Theorem 3.2 (see (61)) imply that

$$
u(x, t)=\inf _{v \in \mathcal{V}}\left\{J_{x t}(v, \tau)+\mathbb{E} u\left(y_{x t}^{v}(t+\tau), t+\tau\right)\right\} .
$$


Corollary 3.4. The dynamic programming property in the weak sense holds (see Definition 1.1) and hence the value function satisfies (33).

Denote

$$
\begin{aligned}
A u(x, t)= & \frac{-\partial u(x, t)}{\partial t}-\frac{1}{2} \beta(t) \circ D^{2} u(x, t)-(a(t) x+b(t)) \circ D u(x, t) \\
& +\alpha(t) u(x, t),
\end{aligned}
$$

where $\circ$ denotes the scalar product of vectors and matrices respectively.

Theorem 3.5 (The HJB equation). The value function u satisfies almost everywhere (a.e.) the following second-order differential equation:

$$
\max \{A u(x, t)-f(x, t),|D u(x, t)|-c(t)\}=0 .
$$

Proof. An application of the DPP for regular stochastic control problems yields for $\epsilon>0$ the following equation (see [5], Chapter IV.3):

$$
A u_{\epsilon}(x, t)+\frac{1}{\epsilon}\left(\left|D u_{\epsilon}(x, t)\right|-c(t)\right)^{+}=f(x, t) \quad \text { a.e. }
$$

In view of Theorems 2.1, 2.2, Remark 2.4, Theorem 3.2, Corollary 3.4 and the Arzela-Ascoli's theorem ([7], Th. 2.4.9) we see that $u_{\epsilon} \rightarrow u$ uniformly on every compact set if $\epsilon \rightarrow 0^{+}$.

Fix $t \in[0, T]$. From (31) and Remark 2.4 we see that $D^{2} u_{\epsilon}(\cdot, t)$ are locally uniformly bounded for all $\epsilon>0$ in their domains, so using the Arzela-Ascoli's theorem from every sequence $\left\{\epsilon_{m}\right\}_{m \in \mathbb{N}}$ convergent to 0 , we can choose a subsequence $\left\{\tilde{\epsilon}_{m}\right\}_{m \in \mathbb{N}}$ such that

$$
D u_{\tilde{\epsilon}_{m}}(\cdot, t) \rightarrow v=\left(v_{1}, \ldots, v_{n}\right) \text { almost uniformly if } m \rightarrow \infty .
$$

But $v$ must be equal to $D u(\cdot, t)$ in the distribution sense. Indeed, for any function $\phi \in C_{c}^{\infty}\left(\mathbb{R}^{n}\right)$ and any $k=1, \ldots, n$ we have

$$
\int_{\mathbb{R}^{n}} \frac{\partial \phi(x)}{\partial x_{k}} u_{\tilde{\epsilon}_{m}}(x, t) d x=-\int_{\mathbb{R}^{n}} \phi(x) \frac{\partial u_{\tilde{\epsilon}_{m}}(x, t)}{\partial x_{k}} d x .
$$

Letting $m \rightarrow \infty$, we get

$$
\int_{\mathbb{R}^{n}} \frac{\partial \phi(x)}{\partial x_{k}} u(x, t) d x=-\int_{\mathbb{R}^{n}} \phi(x) v_{k}(x) d x,
$$

so $v_{k}(\cdot)=\frac{\partial u(\cdot, t)}{\partial x_{k}}$ almost everywhere. Since $\frac{\partial u}{\partial x_{k}}$ and $v_{k}$ are Lipschitz continuous, the equality holds for all $x \in \mathbb{R}^{n}$. Thus, $v$ does not depend on the choice of the subsequence $\left\{\tilde{\epsilon}_{m}\right\}$, so

$$
\forall_{t \in[0, T]} \quad D u_{\epsilon}(\cdot, t) \rightarrow D_{u}(\cdot, t) \quad \text { almost uniformly if } \epsilon \rightarrow 0^{+} .
$$


Let $\psi=(1+|x|)^{-2 p-n-1}$. From (29)-(33) we conclude that $\left|A u_{\epsilon}(x, t)\right|$ is not greater than

$$
\begin{aligned}
\left(C_{33}+C_{29} \alpha_{\max }\right)\left(1+|x|^{p}\right) & +\frac{1}{2} \beta_{\max } n^{2} C_{31}(1+|x|)^{(p-2)^{+}} \\
& +\left(a_{\max }|x|+b_{\max }\right) n C_{30}\left(1+2|x|^{p-1}\right)
\end{aligned}
$$

for almost every $(x, t) \in \mathbb{R}^{n} \times[0, T]$. Using Lemma 1.2, we have the estimate

$$
\left|A u_{\epsilon}(x, t)\right| \leq C_{68}(1+|x|)^{p} \quad \text { a.e. }
$$

for some constant $C_{68}>0$ depending only on $C_{29}, C_{30}, C_{31}, C_{33}, n, p, a_{\max }$, $b_{\max }, \alpha_{\max }, \beta_{\max }$. Hence

$$
\left|A u_{\epsilon}(x, t)\right|^{2} \psi(x) \leq \frac{C_{68}^{2}(1+|x|)^{2 p}}{(1+|x|)^{2 p+n+1}}=\frac{C_{68}^{2}}{(1+|x|)^{n+1}} \quad \text { a.e. }
$$

The same estimate holds for $u$ instead $u_{\epsilon}$. So $\left|A u_{\epsilon}\right|^{2} \psi,|A u|^{2} \psi \in L^{1}\left(\mathbb{R}^{n} \times\right.$ $[0, T])$. Moreover, $A u_{\epsilon}, A u$ are uniformly bounded in the space $L_{\psi}^{2}$, where

$$
L_{\psi}^{2}=\left\{v: v^{2} \psi \in L^{1}\left(\mathbb{R}^{n} \times[0, T]\right)\right\}=L_{\psi \cdot \mu_{L e b}}^{2}\left(\mathbb{R}^{n} \times[0, T]\right) .
$$

From the Banach-Alaoglu theorem ([12], Th. 3.15) we know that balls in the space $L^{2}$ are weakly compact. So for each sequence $\left\{\epsilon_{m}\right\}_{m \in \mathbb{N}}$ convergent to 0 , there exists a subsequence $\left\{\tilde{\epsilon}_{m}\right\}_{m \in \mathbb{N}}$ such that $A u_{\tilde{\epsilon}_{m}} \rightarrow v$ in $L_{\psi}^{2}$ if $m \rightarrow \infty$. We will show that $v=A u$ in the distribution sense. Indeed, for any function $\phi$ belonging to the class $C_{c}^{\infty}\left(\mathbb{R}^{n} \times[0, T]\right)$, we have

$$
\begin{aligned}
& \int_{0}^{T} \int_{\mathbb{R}^{n}}\left(A u_{\tilde{\epsilon}_{m}}\right) \phi d x d t=\int_{0}^{T} \int_{\mathbb{R}^{n}} \frac{\partial \phi}{\partial t} u_{\tilde{\epsilon}_{m}} d x d t-\frac{1}{2} \int_{0}^{T} \int_{\mathbb{R}^{n}}\left(\beta(t) \circ D^{2} \phi\right) u_{\tilde{\epsilon}_{m}} d x d t \\
& \quad+\int_{0}^{T} \int_{\mathbb{R}^{n}}((a(t) x+b(t)) \circ D \phi) u_{\tilde{\epsilon}_{m}} d x d t+\int_{0}^{T} \int_{\mathbb{R}^{n}} \operatorname{tr}(a(t)) u_{\tilde{\epsilon}_{m}} \phi d x d t \\
& \quad+\int_{0}^{T} \int_{\mathbb{R}^{n}} \alpha(t) \phi u_{\tilde{\epsilon}_{m}} d x d t .
\end{aligned}
$$

Letting $m \rightarrow \infty$, we get

$$
\begin{aligned}
\int_{0}^{T} \int_{\mathbb{R}^{n}} v & \phi d x d t=\int_{0}^{T} \int_{\mathbb{R}^{n}} \frac{\partial \phi}{\partial t} u d x d t-\frac{1}{2} \int_{0}^{T} \int_{\mathbb{R}^{n}}\left(\beta(t) \circ D^{2} \phi\right) u d x d t \\
& +\int_{0}^{T} \int_{\mathbb{R}^{n}}((a(t) x+b(t)) \circ D \phi) u d x d t+\int_{0}^{T} \int_{\mathbb{R}^{n}} \operatorname{tr}(a(t)) u \phi d x d t \\
& +\int_{0}^{T} \int_{\mathbb{R}^{n}} \alpha(t) \phi u d x d t=\int_{0}^{T} \int_{\mathbb{R}^{n}}(A u) \phi d x d t .
\end{aligned}
$$

Hence $A u_{\tilde{\epsilon}_{m}} \rightarrow A u$ in $L_{\psi}^{2}$ if $m \rightarrow \infty$. From uniqueness of the limit we conclude

$$
A u_{\epsilon} \rightarrow A u \quad \text { in } L_{\psi}^{2} \text { if } \epsilon \rightarrow 0^{+}
$$


In view of (66) we have $A u_{\epsilon} \leq f$ a.e. From this and (69) we see that $A u(x, t) \leq f(x, t)$ a.e. This, together with Remark 2.8 ensure us that

$$
\max \{A u(x, t)-f(x, t),|D u(x, t)|-c(t)\} \leq 0 \quad \text { a.e. }
$$

Take a sequence $\epsilon_{n} \rightarrow 0$ and let $\mathcal{D}$ be the set of $(x, t) \in \mathbb{R}^{n} \times[0, T]$ such that (66) holds at $(x, t)$ for all $\epsilon_{n}$. Then $\mu_{L e b}\left(\left(\mathbb{R}^{n} \times[0, T]\right) \backslash \mathcal{D}\right)=0$. Choose $t \in[0, T]$ such that for almost every $x \in \mathbb{R}^{n}$ we have $(x, t) \in \mathcal{D}$. Since $\left|D u_{\epsilon_{n}}(x, t)\right| \rightarrow|D u(x, t)|$ as $n \rightarrow \infty$ (see $\left.(67)\right), \mathbb{I}_{\left\{\left|D u_{\epsilon_{n}}(x, t)\right|<c(t)\right\}}=$ $\mathbb{I}_{\{|D u(x, t)|<c(t)\}}$ for $n$ large enough (depending on $(x, t)$ ). We have from this and (66) that

$$
\mathbb{I}_{\{|D u(x, t)|<c(t)\}} A u_{\epsilon_{n}}(x, t) \rightarrow \mathbb{I}_{\{|D u(x, t)|<c(t)\}} f(x, t) \text { a.e. }
$$

On the other hand, (69) yields

$$
\mathbb{I}_{\{|D u(x, t)|<c(t)\}} A u_{\epsilon_{n}}(x, t) \rightarrow \mathbb{I}_{\{|D u(x, t)|<c(t)\}} A u(x, t) \quad \text { in } L_{\psi}^{2},
$$

so the sequence $\left\{\mathbb{I}_{\{|D u(x, t)|<c(t)\}} A u_{\epsilon_{n}}(x, t)\right\}$ is bounded in $L_{\psi}^{2}$ and thus it is uniformly integrable in $L_{\psi}^{1}$. This, together with (71), implies that for every $\phi \in C_{c}^{\infty}\left(\mathbb{R}^{n} \times[0, T]\right) \subset L_{\psi}^{2}$,

$$
\begin{aligned}
\int_{0}^{T} \int_{\mathbb{R}^{n}} \mathbb{I}_{\{|D u(x, t)|<c(t)\}} & \left(A u_{\epsilon_{n}} \phi \psi\right)(x, t) d x d t \\
& \rightarrow \int_{0}^{T} \int_{\mathbb{R}^{n}} \mathbb{I}_{\{|D u(x, t)|<c(t)\}}(f \phi \psi)(x, t) d x d t,
\end{aligned}
$$

which, together with (72) implies that

$$
\mathbb{I}_{\{|D u(x, t)|<c(t)\}} A u(x, t)=\mathbb{I}_{\{|D u(x, t)|<c(t)\}} f(x, t) \text { a.e. }
$$

4. Existence and uniqueness of the optimal control. The results of this section are analogous to Theorems 7 and 8 from [11].

Fix $(t, x) \in[0, T) \times \mathbb{R}^{n}$ (for $t=T$ the only admissible control is $v(0)=0$ a.s.). Let $m_{t}$ be the measure on $([t, T] \times \Omega, \mathcal{B}([t, T]) \otimes \mathcal{F})$ equal to the product of the Lebesgue's measure and $P$.

Remark 4.1. If a process $X$ is a modification of a process $Y$ and both processes have left-continuous sample paths a.s., then the processes $X, Y$ are indistinguishable (compare Problem 1.1.5, [7]).

Theorem 4.2. The optimal control $v^{*} \in \mathcal{V}$, if it exists, is unique up to the indistinguishability.

Proof. Suppose there are $v_{1}, v_{2} \in \mathcal{V}$ for which $u(x, t)=J_{x t}\left(v_{1}\right)=J_{x t}\left(v_{2}\right)$. Put $v_{0}=\left(v_{1}+v_{2}\right) / 2$. Of course $v_{0} \in \mathcal{V}$. From Lemma 1.7 we have

$$
u(x, t)-J_{x t}\left(v_{0}\right)=\frac{1}{2}\left(J_{x t}\left(v_{1}\right)+J_{x t}\left(v_{2}\right)\right)-J_{x t}(v) \geq 0 .
$$


Let $y_{x t}^{0}, y_{x t}^{1}, y_{x t}^{2}$ be the solutions of (1) corresponding to $v=v_{0}, v_{1}, v_{2}$ respectively. In view of the proof of Lemma 1.7 and strict convexity of the running cost function $f$, we have

$$
f\left(y_{x t}^{0}(s), s\right)<\frac{1}{2} f\left(y_{x t}^{1}(s), s\right)+\frac{1}{2} f\left(y_{x t}^{2}(s), s\right)
$$

provided that $y_{x t}^{1}(s) \neq y_{x t}^{2}(s)$.

Assume that $v_{1}, v_{2}$ are not indistinguishable. Then there exists $s^{\prime} \in(t, T]$ such that $P(A)>0$, where $A=\left\{v_{1}\left(s^{\prime}\right) \neq v_{2}\left(s^{\prime}\right)\right\}$ (see Remark 4.1). Because $v_{1}, v_{2}$ have left-continuous sample paths a.s., there exists $s^{\prime \prime}(\omega) \in\left(t, s^{\prime}\right)$ such that $v_{1}(s) \neq v_{2}(s)$ for all $s \in\left[s^{\prime \prime}(\omega), s^{\prime}\right], \omega \in A$. Thus, $y_{x t}^{1}(s) \neq y_{x t}^{2}(s)$ on some $m_{t}$-nonzero set. This fact together with (75) and the definition of $J_{x t}$ imply that the inequality (74) is strict, so we get a contradiction. We conclude that $v_{1}, v_{2}$ must be indistinguishable.

Lemma 4.3. Let $\left\{z_{n}\right\}_{n \in \mathbb{N}}$ be a sequence in $L^{p}\left(m_{t}\right)$. If $z_{n} \rightarrow 0$ in $L^{p}\left(m_{t}\right)$, then $\mathcal{T} z_{n} \rightarrow 0$ in $L^{p}\left(m_{t}\right)$, where

$$
\mathcal{T} z_{n}(s, \omega)=z_{n}(s, \omega)-\int_{t}^{s} a(r) z_{n}(r, \omega) d r .
$$

Proof. By the Hölder's inequality, the function $g(s, \omega)=\int_{t}^{s} a(r) z_{n}(r, \omega) d r$ satisfies

$$
\|g\|_{L^{p}}^{p} \leq a_{\max }^{p} \mathbb{E} \int_{t}^{T} \int_{t}^{T}\left|z_{n}(r, \omega)\right|^{p} \cdot T^{p / q} d r d s \leq a_{\max }^{p} \cdot T^{1+p / q} \cdot\left\|z_{n}\right\|_{L^{p}}^{p},
$$

so $\mathcal{T}$ is a bounded operator from $L^{p}\left(m_{t}\right)$ into $L^{p}\left(m_{t}\right)$.

Theorem 4.4. There exists an optimal control $v^{*} \in \mathcal{V}$.

Proof. Let $\left\{v_{k}\right\}_{k \in \mathbb{N}}$ be a sequence of admissible controls such that $J_{x t}\left(v_{k}\right) \rightarrow$ $u(x, t)$ as $k \rightarrow \infty$ and let $y_{x t}^{k}$ be the solution of (1) corresponding to $v=v_{k}$. Then $J_{x t}\left(v_{k}\right)$ are uniformly bounded in $k$. By Lemma 1.9 the sequence $\left\{y_{x t}^{k}\right\}_{k \in \mathbb{N}}$ is bounded in $L^{p}\left(m_{t}\right)$ and hence, by the Banach-Alaoglu theorem ([12], Th. 3.15), there exists a subsequence (still denoted by $\left\{y_{x t}^{k}\right\}$ ) and a process $y_{x t}$ such that $y_{x t}^{k} \rightarrow y_{x t}$ in $L^{p}\left(m_{t}\right)$.

Fix $k \in \mathbb{N}$. Since the sequence $\left\{y_{x t}^{i}\right\}_{i \geq k}$ is also convergent to $y_{x t}$, by the Mazur theorem there exists

$$
z_{x t}^{k}=\sum_{i=k}^{n(k)} \alpha_{k, i} \cdot y_{x t}^{i}, \quad \alpha_{k, i} \geq 0, \sum_{i=k}^{n(k)} \alpha_{k, i}=1, k \leq n(k)<\infty
$$

such that $\left\|z_{x t}^{k}-y_{x t}\right\|_{L^{p}} \leq 1 / k$. In particular $z_{x t}^{k} \rightarrow y_{x t}$ in $L^{p}\left(m_{t}\right)$. Let $\eta_{k}=\sum_{i=k}^{n(k)} \alpha_{k, i} \cdot v_{i}$ be the control corresponding to $z_{x t}^{k}$ in (1). Then $\eta_{k} \in \mathcal{V}$. Moreover by Lemma 1.7,

$$
u(x, t) \leq J_{x t}\left(\eta_{k}\right) \leq \sum_{i=k}^{n(k)} \alpha_{k, i} \cdot J_{x t}\left(v_{i}\right) \leq \max _{i=k, \ldots, n(k)} J_{x t}\left(v_{i}\right) \stackrel{k \rightarrow \infty}{\longrightarrow} u(x, t) .
$$


For $s \in[t, T]$ we have

$$
z_{x t}^{k}(s)-z_{x t}^{m}(s)-\int_{t}^{s} a(r)\left(z_{x t}^{k}(r)-z_{x t}^{m}(r)\right) d r=\eta_{k}(s-t)-\eta_{m}(s-t) .
$$

Because $\left\{z_{x t}^{k}\right\}$ is convergent in $L^{p}\left(m_{t}\right), z_{x t}^{k}-z_{x t}^{m}$ goes to 0 in $L^{p}\left(m_{t}\right)$ as $k, m \rightarrow \infty$. Using Lemma 4.3 we conclude that $\left\{\eta_{k}(\cdot-t, \cdot)\right\}_{k \in \mathbb{N}}$ is a Cauchy sequence in $L^{p}\left(m_{t}\right)$ so it is convergent to a process $v \in L^{p}\left(m_{t}\right)$. Without loss of generality we may assume that $v(0) \equiv 0$.

Now we choose a subsequence (still denoted by $k$ ) such that $\eta_{k}(s, \omega) \rightarrow$ $v(s, \omega)$ as $k \rightarrow \infty$ for $(s, \omega) \in \mathcal{A}$, where $\left(\mu_{L e b} \times P\right)(\mathcal{A})=T-t$. For $\omega \in \Omega$ and $s \in[0, T-t]$, we define

$$
\mathcal{A}_{\omega}=\{s \in[0, T-t]:(s, \omega) \in \mathcal{A}\}, \quad \mathcal{A}_{s}=\{\omega \in \Omega:(s, \omega) \in \mathcal{A}\} .
$$

Note that $P\left(\mathcal{A}_{0}\right)=1$ because $\eta_{k}(0)=v(0)=0 P$-a.s. Furthermore, let

$$
\tilde{\Omega}=\left\{\omega \in \Omega: \mu_{L e b}\left(\mathcal{A}_{\omega}\right)=T-t\right\}, \quad \mathcal{S}=\left\{s \in[0, T-t]: P\left(\mathcal{A}_{s}\right)=1\right\} .
$$

Then $P(\tilde{\Omega})=1$ and $\mu_{\text {Leb }}(\mathcal{S})=T-t$. Let $\mathcal{N}$ be a countable subset of $\mathcal{S}$, dense in $[0, T-t]$, including 0 and let $\mathcal{A}_{\mathcal{N}}=\bigcap_{s \in \mathcal{N}} \mathcal{A}_{s}$. We have $P\left(\mathcal{A}_{\mathcal{N}}\right)=1$.

Let $\xi_{k}(s)$ denote the total variation of $\eta_{k}$ on the interval $[0, s]$. Because $J_{x t}\left(\eta_{k}\right)$ are uniformly bounded in $k$, there exists a constant $C>0$ such that $\mathbb{E} \xi_{k}(T-t) \leq C$ for all $k \in \mathbb{N}$. In view of the Fatou's lemma, $\mathbb{E} \liminf \operatorname{in}_{k \rightarrow \infty} \xi_{k}(T-t) \leq \liminf _{k \rightarrow \infty} \mathbb{E} \xi_{k}(T-t) \leq C, \operatorname{so~} \liminf _{k \rightarrow \infty} \xi_{k}(T-t)$ is finite a.s.

Fix $\omega \in \Omega$ and let $\Pi \subset \mathcal{A}_{\omega}, \Pi=\left\{t_{0}, t_{1}, \ldots, t_{m}\right\}, 0=t_{0}<t_{1}<\cdots<$ $t_{m} \leq T-t$. Let $k_{n}=k_{n}(\omega) \rightarrow \infty$ be a sequence of natural numbers such that $\lim _{k_{n} \rightarrow \infty} \xi_{k_{n}}(T-t)=\liminf _{k \rightarrow \infty} \xi_{k}(T-t)$. Then

$$
\begin{aligned}
\sum_{i=0}^{m-1}\left|v\left(t_{i+1}\right)-v\left(t_{i}\right)\right| & =\lim _{k_{n} \rightarrow \infty} \sum_{i=0}^{m-1}\left|\eta_{k_{n}}\left(t_{i+1}\right)-\eta_{k_{n}}\left(t_{i}\right)\right| \\
& \leq \lim _{k_{n} \rightarrow \infty} \xi_{k_{n}}(T-t)=\liminf _{k \rightarrow \infty} \xi_{k}(T-t) .
\end{aligned}
$$

Thus, $v_{\mid \mathcal{A}_{\omega}}$ has bounded variation and hence it has left-hand and right-hand limits at each point. Let $v^{*}=0$ on the $P$-zero set $\Omega \backslash\left(\mathcal{A}_{\mathcal{N}} \cap \tilde{\Omega}\right)$. On $\mathcal{A}_{\mathcal{N}} \cap \tilde{\Omega}$ let

$$
v^{*}(s)=\left\{\begin{array}{cl}
0=v(0), & s=0 \\
\lim _{\omega \ni u \uparrow s} v(u)=\lim _{\mathcal{N} \ni u \uparrow s} v(u), & s \in(0, T-t] .
\end{array}\right.
$$

Then $v^{*}$ is progressively measurable, left-continuous and $v^{*}(0)=0$. Moreover, for $\omega \in \mathcal{A}_{\mathcal{N}} \cap \tilde{\Omega}$ and for each partition $\Pi=\left\{t_{0}, t_{1}, \ldots, t_{m}\right\}, 0=t_{0}<$ $t_{1}<\cdots<t_{m} \leq T-t$, we can choose $\left\{t_{i}^{k}\right\}_{k \in \mathbb{N}} \subset \mathcal{A}_{\omega}$ such that $t_{i}^{k} \uparrow t_{i}$ as 
$k \rightarrow \infty, i=1,2, \ldots, m$. Therefore,

$$
\begin{aligned}
\sum_{i=0}^{m-1}\left|v^{*}\left(t_{i+1}\right)-v^{*}\left(t_{i}\right)\right| & =\lim _{k \rightarrow \infty} \sum_{i=0}^{m-1}\left|v\left(t_{i+1}^{k}\right)-v\left(t_{i}^{k}\right)\right| \\
& \leq \operatorname{Var}\left(v,[0, T-t] \cap \mathcal{A}_{\omega}\right) \leq \liminf _{k \rightarrow \infty} \xi_{k}(T-t)
\end{aligned}
$$

so $v^{*}$ has bounded variation. This ensures us that $v^{*} \in \mathcal{V}$.

For $\omega \in \mathcal{A}_{\mathcal{N}} \cap \tilde{\Omega}$, the set $A_{\omega} \cap\left\{s \in[0, T-t]: v(s, \omega) \neq v^{*}(s, \omega)\right\}$ is countable, so its Lebesgue's measure is equal to 0 . Therefore $v=v^{*} m_{t}$-a.e. In particular, $\eta_{k} \rightarrow v^{*}$ in $L^{p}\left(m_{t}\right)$. Proceeding as in Steps 3-4 in the proof of Theorem 3.2 we can show that $y_{x t}^{\eta_{k}} \rightarrow y_{x t}^{v^{*}}$ in $L^{p}\left(m_{t}\right)$ and hence

$$
\lim _{k \rightarrow \infty} \mathbb{E} \int_{t}^{T} f\left(y_{x t}^{\eta_{k}}(s), s\right) e^{-\int_{t}^{s} \alpha(r) d r} d s=\mathbb{E} \int_{t}^{T} f\left(y_{x t}^{v^{*}}(s), s\right) e^{-\int_{t}^{s} \alpha(r) d r} d s
$$

To finish the proof we need to check that

$$
\mathbb{E} \int_{t}^{T} c(s) e^{-\int_{t}^{s} \alpha(r) d r} d \xi^{*}(s-t) \leq \liminf _{k \rightarrow \infty} \mathbb{E} \int_{t}^{T} c(s) e^{-\int_{t}^{s} \alpha(r) d r} d \xi_{k}(s-t),
$$

where $\xi^{*}$ is the total variation of $v^{*}$. Fix $\omega \in \mathcal{A}_{\mathcal{N}} \cap \tilde{\Omega}$ and let $0 \leq s_{1} \leq$ $s_{2} \leq T-t$. Let $\Pi=\left\{t_{0}, t_{1}, \ldots, t_{m}\right\}, s_{1}=t_{0}<t_{1}<\cdots<t_{m}=s_{2}$ and let $\left\{t_{i}^{k}\right\}_{k \in \mathbb{N}} \subset \mathcal{A}_{\omega}$ be such that $t_{i}^{k} \uparrow t_{i}$ as $k \rightarrow \infty, i=0,1, \ldots, m$. Then for every $k_{0} \in \mathbb{N}$

$$
\begin{aligned}
\sum_{i=0}^{m-1}\left|v^{*}\left(t_{i+1}\right)-v^{*}\left(t_{i}\right)\right| & =\lim _{k \rightarrow \infty} \sum_{i=0}^{m-1}\left|v\left(t_{i+1}^{k}\right)-v\left(t_{i}^{k}\right)\right| \\
& \leq \operatorname{Var}\left(v,\left[t_{0}^{k_{0}}, s_{2}\right] \cap \mathcal{A}_{\omega}\right) .
\end{aligned}
$$

Letting $k_{0} \rightarrow \infty$, we get $\sum_{i=0}^{m-1}\left|v^{*}\left(t_{i+1}\right)-v^{*}\left(t_{i}\right)\right| \leq \operatorname{Var}\left(v,\left[s_{1}, s_{2}\right] \cap \mathcal{A}_{\omega}\right)$ and hence

$$
\operatorname{Var}\left(v^{*},\left[s_{1}, s_{2}\right]\right) \leq \operatorname{Var}\left(v,\left[s_{1}, s_{2}\right] \cap \mathcal{A}_{\omega}\right) .
$$

Let $\xi(s)=\operatorname{Var}\left(v,[0, s] \cap \mathcal{A}_{\omega}\right), s \in[0, T-t]$. Restricting $\Pi=\left\{t_{0}, t_{1}, \ldots, t_{m}\right\}$, $s_{1}=t_{0}<t_{1}<\cdots<t_{m}=s_{2}$ so that $\Pi \subset \mathcal{A}_{\omega}$ (in particular assuming $\left.s_{1}, s_{2} \in \mathcal{A}_{\omega}\right)$, we get

$$
\begin{aligned}
\sum_{i=0}^{m-1}\left|v\left(t_{i+1}\right)-v\left(t_{i}\right)\right| & =\lim _{k \rightarrow \infty} \sum_{i=0}^{m-1}\left|\eta_{k}\left(t_{i+1}\right)-\eta_{k}\left(t_{i}\right)\right| \\
& \leq \liminf _{k \rightarrow \infty}\left(\xi_{k}\left(s_{2}\right)-\xi_{k}\left(s_{1}\right)\right) .
\end{aligned}
$$

As $\|\Pi\| \rightarrow 0$ we get

$$
\xi\left(s_{2}\right)-\xi\left(s_{1}\right) \leq \liminf _{k \rightarrow \infty}\left(\xi_{k}\left(s_{2}\right)-\xi_{k}\left(s_{1}\right)\right) .
$$

Now take $\Pi=\left\{t_{0}, t_{1}, \ldots, t_{m}\right\}, 0=t_{0}<t_{1}<\cdots<t_{m} \leq T-t, \Pi \subset$ $\mathcal{N}$. In particular, $\Pi \subset \mathcal{A}_{\omega}$ for all $\omega \in \mathcal{A}_{\mathcal{N}}$. For every interval $\left[t_{i}, t_{i+1}\right]$, 
$i=0,1, \ldots, m-1$, let $l_{i}=\min \left\{c(s) e^{-\int_{t}^{s} \alpha(r) d r}: s \in\left[t_{i}+t, t_{i+1}+t\right]\right\}$. For $\omega \in \mathcal{A}_{\mathcal{N}} \cap \tilde{\Omega}$, by $(78)-(79)$, we have

$$
\begin{aligned}
\sum_{i=0}^{m-1} l_{i} \cdot\left(\xi^{*}\left(t_{i+1}\right)-\xi^{*}\left(t_{i}\right)\right) & \leq \sum_{i=0}^{m-1} l_{i} \cdot\left(\xi\left(t_{i+1}\right)-\xi\left(t_{i}\right)\right) \\
& \leq \liminf _{k \rightarrow \infty} \sum_{i=0}^{m-1} l_{i} \cdot\left(\xi_{k}\left(t_{i+1}\right)-\xi_{k}\left(t_{i}\right)\right) .
\end{aligned}
$$

This together with the Fatou's lemma and the fact that $P\left(\mathcal{A}_{\mathcal{N}} \cap \tilde{\Omega}\right)=1$ yields

$$
\begin{aligned}
\mathbb{E} \sum_{i=0}^{m-1} l_{i} \cdot\left(\xi^{*}\left(t_{i+1}\right)-\xi^{*}\left(t_{i}\right)\right) & \leq \mathbb{E} \liminf _{k \rightarrow \infty} \sum_{i=0}^{m-1} l_{i} \cdot\left(\xi_{k}\left(t_{i+1}\right)-\xi_{k}\left(t_{i}\right)\right) \\
& \leq \liminf _{k \rightarrow \infty} \mathbb{E} \sum_{i=0}^{m-1} l_{i} \cdot\left(\xi_{k}\left(t_{i+1}\right)-\xi_{k}\left(t_{i}\right)\right) \\
& \leq \liminf _{k \rightarrow \infty} \mathbb{E} \int_{t}^{T} c(s) e^{-\int_{t}^{s} \alpha(r) d r} d \xi_{k}(s-t) .
\end{aligned}
$$

Letting $\|\Pi\| \rightarrow 0, t_{m} \uparrow T-t$ so that each partition in the sequence is contained in the next one, by the monotone convergence theorem, we get (77).

From (76) and (77)

$$
J_{x t}\left(v^{*}\right) \leq \liminf _{k \rightarrow \infty} J_{x t}\left(\eta_{k}\right)=u(x, t) .
$$

On the other hand, $J_{x t}\left(v^{*}\right) \geq u(x, t)$ because $v^{*} \in \mathcal{V}$ and hence $J_{x t}\left(v^{*}\right)=$ $u(x, t)$ so $v^{*}$ is an optimal control.

\section{REFERENCES}

[1] Budhiraja, A., Ross, K., Existence of optimal controls for singular control problems with state constraints, Ann. Appl. Probab. 16, No. 4 (2006), 2235-2255.

[2] Chow, P. L., Menaldi, J. L., Robin, M., Additive control of stochastic linear systems with finite horizon, SIAM J. Control Optim. 23, No.6 (1985), 858-899.

[3] Dufour, F., Miller, B., Singular stichastic control problems, SIAM J. Control Optim. 43, No. 2 (2004), 708-730.

[4] Evans, L. C., Partial Differential Equations, American Mathematical Society, Providence, RI, 1998.

[5] Fleming, W. H., Soner, H. M., Controlled Markov Processes and Viscosity Solutions, Springer, New York, 2006.

[6] Haussman, U. G., Suo, W., Singular optimal stochastic controls. I. Existence, SIAM J. Control Optim. 33, No. 3 (1995), 916-936.

[7] Karatzas, I., Shreve, S. E., Brownian Motion and Stochastic Calculus, SpringerVerlag, New York, 1988.

[8] Kruk, Ł., Optimal policies for n-dimensional singular stochastic control problems, Part I: The Skorokhod problem, SIAM J. Control Optim. 38, No. 5 (2000), 16031622. 
[9] Kruk, Ł., Optimal policies for n-dimensional singular stochastic control problems, Part II: The radially symmetric case. Ergodic control, SIAM J. Control Optim. 39, No. 2 (2000), 635-659.

[10] Krylov, N. V., Controlled Diffusion Processes, Springer-Verlag, New York, 1980.

[11] Menaldi, J. L., Taksar, M. I., Optimal correction problem of a multidimensional stochastic system, Automatica J. IFAC 25, No. 2 (1989), 223-232.

[12] Rudin, W., Functional Analysis, McGraw-Hill Book Company, New York, 1991.

[13] Rudin, W., Principles of Mathematical Analysis, McGraw-Hill Book Company, New York, 1976.

[14] Soner, H. M., Shreve, S. E., Regularity of the value function for a two-dimensional singular stochastic control problem, SIAM J. Control Optim. 27 (1989), 876-907.

[15] Soner, H. M., Shreve, S. E., A free boundary problem related to singular stochastic control, Applied stochastic analysis (London, 1989), Stochastics Monogr. 5, Gordon and Breach, New York, 1991, 265-301.

[16] Soner, H. M., Shreve, S. E., A free boundary problem related to singular stochastic control: the parabolic case, Comm. Partial Differential Equations 16 (1991), 373-424.

[17] S. A. Williams, P. L. Chow and J. L. Menaldi, Regularity of the free boundary in singular stochastic control, J. Differential Equations 111 (1994), 175-201.

[18] http://en.wikipedia.org/wiki/Gronwall's_inequality, 24.09.2013.

Marcin Boryc

Institute of Mathematics

Maria Curie-Skłodowska University

pl. M. Curie-Skłodowskiej 1

20-031 Lublin

Poland

e-mail: borycmarcin@gmail.com

Received April 24, 2014
Łukasz Kruk

Institute of Mathematics

Maria Curie-Skłodowska University

pl. M. Curie-Skłodowskiej 1

20-031 Lublin

Poland

e-mail: lkruk@hektor.umcs.lublin.pl 